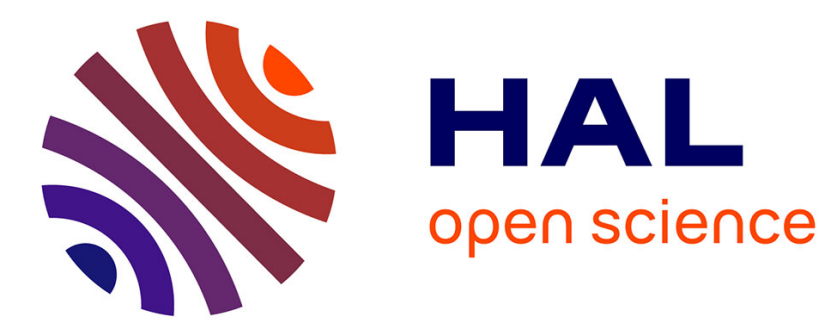

\title{
Comment appliquer la directive nitrates et à quel coût? une modélisation spatiale du marché de l'épandage
}

Fabien Djaout, Philippe Le Goffe, Mélanie Tauber

\section{To cite this version:}

Fabien Djaout, Philippe Le Goffe, Mélanie Tauber. Comment appliquer la directive nitrates et à quel coût? une modélisation spatiale du marché de l'épandage. 2008. hal-01594064

\author{
HAL Id: hal-01594064 \\ https://hal.science/hal-01594064
}

Preprint submitted on 26 Sep 2017

HAL is a multi-disciplinary open access archive for the deposit and dissemination of scientific research documents, whether they are published or not. The documents may come from teaching and research institutions in France or abroad, or from public or private research centers.
L'archive ouverte pluridisciplinaire HAL, est destinée au dépôt et à la diffusion de documents scientifiques de niveau recherche, publiés ou non, émanant des établissements d'enseignement et de recherche français ou étrangers, des laboratoires publics ou privés. 
Comment appliquer la directive nitrates et à quel coût ? Une modélisation spatiale du marché de l'épandage

\author{
Fabien DJAOUT, Philippe LE GOFFE, Mélanie TAUBER
}

Working Paper SMART - LERECO N08-02

June 2008 


\title{
Comment appliquer la directive nitrates et à quel coût ? Une modélisation spatiale du marché de l'épandage
}

\author{
Fabien DJAOUT \\ Ministère de l'écologie et du développement durable (MEDD), Direction des \\ études économiques et de l'évaluation environnementale (D4E), F-75007 Paris, France (lors \\ de ce travail) \\ Philippe LE GOFFE \\ Agrocampus Ouest, UMR1302 SMART, F-35000 Rennes, France \\ INRA, UMR1302 SMART, F-35000 Rennes, France \\ Mélanie TAUBER \\ Ministère de l'écologie et du développement durable (MEDD), Direction des \\ études économiques et de l'évaluation environnementale (D4E), F-75007 Paris, France
}

Le travail a été réalisé au Ministère de l'écologie et du développement durable (MEDD), Direction des études économiques et de l'évaluation environnementale (D4E). Les auteurs remercient Sylvain Maestracci pour sa participation au comité de pilotage de l'étude.

\section{Auteur pour la correspondance / Corresponding author}

\section{Philippe LE GOFFE}

UMR SMART - Agrocampus Ouest

65 rue de Saint-Brieuc, CS 84215

35042 Rennes cedex, France

Email: Philippe.Legoffe@agrocampus-ouest.fr

Téléphone / Phone: +33 (0)2 23485416

Fax: +33 (0)2 23485417 


\title{
Comment appliquer la directive nitrates et à quel coût ? \\ Une modélisation spatiale du marché de l'épandage
}

\section{Résumé}

En Bretagne, la concentration des élevages est responsable de la pollution de l'eau. La politique française de l'eau n'est pas conforme à la directive européenne, à la différence des pays du nord où le principe pollueur-payeur a stimulé les marchés de l'épandage. Notre objectif était de chiffrer le coût de la directive nitrates et de simuler le fonctionnement des marchés de l'épandage en Bretagne. Un modèle linéaire minimise le coût de gestion des effluents d'élevage sous contrainte de la directive nitrates. Les élevages traitent ou exportent leurs effluents à court terme et réduisent leur cheptel à long terme, de 20 à $40 \%$ selon les espèces. Le marché procure des économies importantes par rapport à l'absence d'échanges ou un plan de maintien de l'élevage.

Mots-clés : effluents d'élevage, directive nitrates, marché, modèle linéaire

Classification JEL : Q0, Q2

\section{The cost of the Nitrate Directive: a spatial model of manure market}

\begin{abstract}
In Brittany, animal concentration is responsible for water pollution. French water policy does not stick to Water Framework Directive, though in northern countries polluter-pay principle stimulated manure markets. We tried to calculate the cost of the Nitrate Directive and simulate the manure markets in Brittany. A linear model minimizes the cost of manure management constrained by the Nitrate Directive. Farms process or export their manure in the short-run and reduce their livestock in the long-run, by 20 to $40 \%$ according to types. Market leads to significant savings compared to no trade or to a regional plan aiming at maintaining livestock production.
\end{abstract}

Keywords: manure, nitrate directive, market, linear model

JEL Classification: Q0, Q2 


\section{Comment appliquer la directive nitrates et à quel coût ? \\ Une modélisation spatiale du marché de l'épandage}

\section{Introduction}

La Bretagne est un des grands bassins de production animale intensive en Europe, au même titre que les Pays-Bas, le Danemark ou la Catalogne en Espagne. Dans cette région, la densité animale élevée est à l'origine d'une pollution de l'eau, qui a valu à la France d'être condamnée à plusieurs reprises par la Cour européenne de justice, pour non respect des directives sur l'eau potable. Un contentieux en cours portant sur plusieurs bassins versants bretons prévoit une amende et des astreintes lourdes, dans le cas où les mesures proposées par la France ne seraient pas jugées suffisantes par la Commission européenne. En France, la politique de maîtrise des pollutions des élevages repose surtout sur la réglementation et les subventions, la formation et le volontariat des acteurs. Un des instruments principaux de l'application française de la directive européenne sur les nitrates est l'obligation de traitement des effluents au dessus de plafonds d'épandage et son subventionnement par l'Etat et les collectivités. En outre, la loi sur l'eau récemment votée prévoit que l'ancienne redevance pollution des élevages, dont le taux était déjà très faible, soit désormais forfaitaire. Ceci explique que les agriculteurs français contribuent très peu au budget des Agences de l'eau. Cette politique n'est pas cohérente avec les principes économiques de la Directive cadre européenne sur l'eau, qui prônent le Principe pollueur-payeur, la récupération des coûts par secteur économique et la minimisation des coûts (Le Goffe, 2005).

Ce traitement de la pollution des élevages par la réglementation et les aides n'est pas l'apanage de la France. De nombreux pays, comme par exemple les Etats-Unis et la province canadienne du Québec en Amérique du nord, l'Espagne en Europe, ont une politique similaire. En revanche le Danemark et les Pays-Bas, confrontés il est vrai à des charges animales plus élevées, ont privilégié le Principe pollueur-payeur et les incitations économiques. Les dépassements des normes de fertilisation de la directive nitrates sont lourdement pénalisés et le traitement des effluents n'est pas aidé par l'Etat. Cela a incité les éleveurs de ces pays à optimiser l'épandage, via le développement d'un marché organisé par les coopératives et les négociants, et à réduire leur cheptel aux Pays-Bas.

Ce n'est pas le cas en Bretagne, où on estime que l'épandage chez les tiers représente seulement la moitié de la surface d'épandage potentielle qui pourrait être mise à la disposition 
des éleveurs par les agriculteurs. Cette situation est d'abord due au manque d'incitations économiques pour les demandeurs et les offreurs potentiels de terres d'épandage. D'une part les excédents de minéraux ne sont pas systématiquement pénalisés et la législation sur les épandages ne s'applique pas avec la rigueur nécessaire (Baron et al., 2001), d'autre part le traitement des effluents est partiellement subventionné. Cela n'incite pas les éleveurs à rechercher des terres d'épandage, même si il est moins coûteux de couvrir les coûts éventuels des prêteurs de terre, liés au remplacement des engrais minéraux par des amendements organiques, que de recourir au traitement des effluents ou à la réduction du cheptel. Une autre raison, liée à la précédente, est que l'organisation insuffisante du marché de l'épandage peut être à l'origine de coûts de transaction qui freinent les échanges. En effet, il n'existe pas de structure qui centralise les offres et les demandes de terre d'épandage, même si les coopératives et les fabricants d'aliments peuvent jouer ce rôle de manière informelle. Enfin, il ne faut pas sous-estimer l'opposition des riverains aux transferts d'effluents.

Cette difficulté à mettre en œuvre des politiques efficaces vient notamment de ce que l'application de contraintes environnementales dans les zones à forte concentration animale est à l'origine de la croissance des coûts de gestion des effluents d'élevage. Ces coûts remettent en question la concentration spatiale des filières animales basée sur des économies d'agglomération. Des modèles économiques régionaux, reposant sur la maximisation des profits ou la minimisation des coûts sous contrainte de normes d'épandage des effluents, ont été utilisés pour chiffrer ces coûts. En Europe, Helming (1998) et Reyns et al. (1999) ont simulé respectivement l'impact de taxes sur l'azote ou de scénarios de politique du lisier sur les coûts de gestion et l'utilisation des effluents, aux Pays-Bas et dans les Flandres belges. Cependant, en modélisant les échanges d'effluents entre quelques grandes régions, ces travaux ne prennent pas assez finement en compte la dimension spatiale du problème du lisier. En Amérique, des études voisines ont mis en évidence les pertes de bien-être des producteurs liées aux coûts de transport (voir notamment Ribaudo et al., 2004) et à la substitution limitée de l'azote organique à l'azote minéral (Kaplan et al., 2004). A une échelle plus petite, les travaux de Smith et al. (2006) sont originaux, car ils intègrent la distribution spatiale réelle de la production animale et des cultures dans la région et montrent l'intérêt du traitement des effluents (compostage), alors que les études antérieures considéraient seulement l'épandage sur le sol pour résorber les effluents.

Confrontées à la question de savoir à quelle condition les effluents d'élevage sont des ressources ou des déchets, d'autres études ont cherché à mesurer la valeur des effluents 
d'élevage pour les producteurs. Parmi les plus récentes, Keplinger et Hauck (2006) montrent que l'accroissement de la production d'effluents entraîne une augmentation de leur coût de transport et une diminution de leur valeur marginale, qui devient vite négative dans certains cas, d'autant plus que les restrictions d'application sont sévères. Dans ce dernier cas, la concurrence pour les terres d'épandage fait que les effluents deviennent des déchets, dont l'élimination est coûteuse. Feinerman et Komen (2005) se sont intéressés aux demandeurs d'effluents en modélisant la substitution entre les engrais organiques et les engrais minéraux dans la production végétale. Il apparaît qu'en l'absence de subventions, payées notamment par les éleveurs, les agriculteurs hollandais préfèrent apporter l'azote sous forme exclusivement minérale. Ribaudo et al. (2003) suggèrent que les éleveurs puissent payer les agriculteurs, afin d'augmenter leur consentement à accepter des engrais organiques, ce qui permettrait d'éviter des coûts de transport. Certains auteurs ont formalisé cette intuition en proposant une modélisation théorique des marchés de droits d'épandage, dans laquelle les éleveurs ont accès à d'autres solutions de résorption des effluents que le transport et l'épandage. Chez Rainelli et Vermersch (2000), la demande inverse de droits est donnée par le profit marginal de l'éleveur, qui arbitre entre la location de droits et la réduction du cheptel. Le Goffe et Salanié (2005) montrent que le statut des effluents d'élevage - fertilisant valorisé ou déchet coûteux - dépend du bénéfice ou du coût de substitution de l'azote organique à l'azote minéral, de la rentabilité des élevages et du coût de traitement des effluents, c'est-àdire de l'offre et de la demande de droits sur le marché de l'épandage.

Alors que la directive nitrates n'est toujours pas vraiment appliquée en France, l'étude a pour objectif de mesurer le coût de mise en conformité avec cette directive. Il s'agit également de simuler le fonctionnement d'un marché de l'épandage, qui pourrait se développer si la directive nitrates et la directive cadre européenne sur l'eau étaient effectivement appliquées en France. Outre l'analyse des quantités d'effluents échangées, des lieux d'échange et des prix, on souhaitait obtenir les solutions choisies par les éleveurs pour résorber leurs effluents d'élevage et les économies par rapport à une situation sans échanges, ou figée par un plan administratif. Cette problématique est pertinente dans un pays où le marché de l'épandage ne fonctionne pas bien, faute d'application du principe pollueur-payeur. Un modèle linéaire qui minimise les coûts régionaux de gestion des effluents a été construit pour simuler le marché, comme cela a été fait pour les marchés de droits d'eau (Pujol et al., 2006). Il prend en compte la localisation actuelle de la production animale et donc les situations locales de surplus. La contrainte environnementale porte seulement sur l'azote, dans la mesure où la réglementation 
sur le phosphore n'est pas vraiment effective en France, à la différence d'autres pays (PaysBas, Danemark, Canada...). L'étude porte sur le département breton des Côtes d'Armor, qui a la plus forte densité animale de France. L'article présente successivement le cadre microéconomique de l'étude, le modèle linéaire et les données qui ont servi à l'alimenter, les résultats des scénarios testés et termine par une discussion.

\section{Cadre théorique}

Le Goffe et Salanié (2005) ont proposé une modélisation du marché de l'épandage, basée sur les programmes d'un producteur spécialisé en élevage hors-sol et d'un agriculteur spécialisé en production végétale. La maximisation séparée du profit des producteurs conduit à la demande individuelle de droits d'épandage de l'éleveur et à l'offre individuelle de droits de l'agriculteur, ainsi qu'à sa demande dérivée de terre. La demande inverse s'interprète comme le coût marginal de traitement des effluents ou le profit marginal de l'élevage. L'offre inverse s'interprète comme le coût marginal de substitution de l'azote organique à l'azote minéral, qui est probablement négatif pour les premiers kilos d'azote organique substitués (consentement à payer), puis devient positif quand la proportion d'azote organique est importante. L'augmentation de l'azote organique peut donc représenter une économie, par exemple dans les zones spécialisées en culture qui l'utilisent peu, ou un coût pour les zones d'élevage qui en ont beaucoup. Enfin, en agrégeant les fonctions individuelles d'offre et de demande de droits, Le Goffe et Salanié (2005) montrent que le prix d'équilibre du droit d'épandage augmente avec la densité animale.

Alors que le modèle micro-économique que nous venons d'évoquer suppose que les rendements sont décroissants, le choix de la programmation linéaire implique que les rendements et les coûts unitaires sont constants. Cette hypothèse simplificatrice caricature la réalité, car on soupçonne qu'il existe des économies d'échelle dans la production animale et le traitement des effluents, au moins jusqu'à une certaine taille (rendements croissants, puis décroissants). Cependant, mesurer ces économies d'échelle aurait demandé de mener un important travail économétrique d'estimation des fonctions de coût, à partir des données comptables d'un échantillon d'exploitations, dans chaque espèce animale (sachant que les données sur le traitement sont rares, vu le faible nombre d'exploitations équipées). A contrario, la programmation linéaire permet d'obtenir rapidement des résultats, à partir de données réduites de la comptabilité d'exploitations type. Pour atténuer les erreurs dues aux 
rendements constants, nous avons fait varier les marges et les coûts unitaires en considérant, pour chaque type d'élevage, trois classes de taille d'exploitation.

Dans notre modèle linéaire, la demande inverse de droits agrégée adressée à chaque unité spatiale n'est pas une fonction continue, mais une fonction en escalier où chaque marche correspond à un atelier d'élevage de la zone ou d'une autre zone. La largeur et la hauteur d'une marche sont respectivement la production d'azote et le coût unitaire de résorption le plus bas de l'atelier, diminué du coût de transport et d'épandage (ce qui explique que les ateliers trop éloignés de la zone ne demandent pas de droits). L'offre de droits agrégée est rigide, car on n'a pas pris en compte les économies et les coûts liés à l'offre de terres d'épandage, dans la fonction objectif de notre modèle linéaire, faute de données disponibles. A l'équilibre, seuls les ateliers ayant un coût unitaire de résorption net supérieur au prix des droits louent effectivement des droits dans la zone.

Si on ajoute des coûts de transport et d'épandage au modèle micro-économique, on montre que le programme des agents conduit à l'égalité des coûts ou profits marginaux des différents producteurs, nets des coûts de transport et d'épandage, c'est-à-dire la condition de la minimisation du coût global de résorption. En reprenant cette condition dans la fonction objectif, le modèle linéaire permet de simuler le fonctionnement du marché. Cependant, le caractère constant des coûts de résorption unitaires et la décision binaire des ateliers de louer ou de ne pas louer des droits dans les différentes zones empêchent d'obtenir cette égalisation des coûts unitaires nets. L'exportation des effluents n'est pas intéressante lorsque le coût unitaire de résorption de l'atelier exportateur, net du coût de transport et du différentiel de coûts d'épandage entre ateliers, est inférieur au coût unitaire de résorption de l'atelier importateur. On s'attend donc à observer des prix de droits différents selon les zones du territoire, qui simulent des marchés locaux de l'épandage.

\section{Modèle linéaire et données}

Le modèle permet de simuler différents scénarios de gestion des effluents d'élevage dans le département des Côtes d'Armor (Bretagne). Ce département a été choisi en raison de la grande concentration de la production animale, notamment de la production hors-sol (porcs, volailles chair, poules pondeuses). En 2000, la production d'azote organique du département était de 66000 tonnes pour 480000 hectares de surface agricole utile (SAU) et 40 cantons sur 
51 étaient en zone d'excédent structurel $\left(Z^{2} S^{1}\right)$. La production hors-sol représente plus de 50 $\%$ de l'azote organique produit et l'essentiel des excédents d'azote des exploitations. Bien que produisant l'autre moitié de l'azote organique, les bovins génèrent peu d'excédents, car ce sont des productions liées au sol. La production hors-sol est concentrée au centre du département, autour de Saint-Brieuc : porcs à l'est, poules à l'ouest, volailles chair au sud. On y trouve logiquement les cantons en ZES (figure 1), avec des charges maximales de l'ordre de $300 \mathrm{~kg}$ par hectare épandable (canton de Lamballe), alors que les cantons ayant des capacités d'accueil en azote organique sont situés à l'est et surtout à l'ouest du département.

Une modélisation réaliste de la gestion des effluents d'élevage sur un territoire nécessite de prendre en compte la répartition spatiale de la production animale, source d'excédents et de pollution, afin d'introduire les coûts de transport des lisiers et fumiers qui constituent le facteur limitant des échanges. La taille de l'unité spatiale élémentaire a été choisie de manière à pouvoir négliger les coûts de transport à l'intérieur de l'unité, sans trop multiplier le nombre d'unités pour ne pas compliquer la programmation. Le niveau de définition retenu est le canton $^{2}$. Les données cantonales du Recensement général de l'agriculture (RGA) 2000 ont permis d'établir la structure de la production animale et celle du foncier, qui sont fixées. Néanmoins, la réduction du cheptel est une méthode de résorption possible. La production annuelle d'azote organique des élevages a été calculée à partir des effectifs animaux et des normes de rejets azotés établies par le Corpen ${ }^{3}$.

Le modèle distingue 17 ateliers représentatifs, dont l'azote et le foncier sont agrégés au niveau de chaque canton. Le tableau 1 présente une agrégation départementale de la production d'azote organique et de la capacité d'accueil (en azote) des terres en propre pour les 17 ateliers. On compte un atelier de production végétale sans animaux et quatre types d'élevage (porc, volaille chair, poule pondeuse, bovin) divisés en quatre catégories selon la spécialisation (atelier principal ${ }^{4}$, atelier secondaire) et la production d'azote de l'atelier

\footnotetext{
${ }^{1}$ On parle de ZES lorsque la production d'azote organique du canton est supérieure à $170 \mathrm{~kg}$ par hectare de surface épandable, limite fixée par la directive nitrates.

${ }^{2}$ Il est probable que ce choix conduise à minorer les coûts de transport et à majorer les échanges.

${ }^{3}$ Calculs effectués par la Direction Régionale de l’Agriculture et de la Forêt de Bretagne.

${ }^{4}$ L'atelier qui compte plus de $70 \%$ de la production d'azote de l'exploitation est dit principal. Il définit la spécialisation de l'exploitation. Les ateliers de type différent qui produisent le reste de l'azote sont dits secondaires.
} 
principal (moins de $20000 \mathrm{~kg}$, de 20000 à $30000 \mathrm{~kg}$, plus de $30000 \mathrm{~kg}$ ). Cette segmentation a pour objectif de prendre en compte la variation des coûts de résorption selon l'espèce et la taille de l'atelier animal et de permettre de mieux simuler les échanges intra-cantonaux (on ne simule pas les échanges intra-atelier).

Tableau 1. Production d'azote organique et capacité d'accueil ${ }^{1}$ des terres en propre selon le type d'atelier et la classe de taille : agrégation sur le département des Côtes d'Armor (kg d'azote, RGA 2000)

\begin{tabular}{|l|l|l|l|l|l|l|}
\hline \multicolumn{2}{|l|}{ Taille de l'atelier (kg d'azote) } & $<20000$ & 20000 à 30000 & $>30000$ & \multicolumn{1}{c|}{$\begin{array}{c}\text { Atelier } \\
\text { secondaire }\end{array}$} & Total \\
\hline \multirow{2}{*}{ Porcins } & Production & 13354865 & 2423476 & 1155724 & 4329702 & 21263767 \\
\cline { 2 - 7 } & Capacité d'accueil & 8502890 & 933300 & 236980 & 0 & 9673170 \\
\hline \multirow{2}{*}{$\begin{array}{l}\text { Poules } \\
\text { pondeuses }\end{array}$} & Production & 2824322 & 1080290 & 2271587 & 963508 & 7139707 \\
\cline { 2 - 7 } & Capacité d'accueil & 1173000 & 287810 & 209610 & 0 & 1670420 \\
\hline \multirow{2}{*}{$\begin{array}{l}\text { Volailles } \\
\text { chair }\end{array}$} & Production & 3842749 & 321831 & 73853 & 1834398 & 6072831 \\
\cline { 2 - 7 } & Capacité d'accueil & 1411170 & 72420 & 11730 & 0 & 1495320 \\
\hline \multirow{2}{*}{$\begin{array}{l}\text { Bovins } \\
\text { Végétal }\end{array}$} & Production & 25637922 & 221070 & 0 & 5688256 & 31547248 \\
\cline { 2 - 7 } & Capacité d'accueil & 45391700 & 144670 & 0 & 0 & 45536370 \\
\hline
\end{tabular}

${ }^{1} 170 \mathrm{~kg}$ d'azote organique par hectare de surface épandable.

On a besoin de la surface détenue par les ateliers agrégés pour comparer les solutions et les coûts de résorption, selon qu'on autorise ou pas les transferts d'effluents, et ainsi chiffrer l'intérêt économique du marché. Le RGA ne donne pas d'éléments sur la façon dont la surface des exploitations est affectée à l'épandage des ateliers principaux ou secondaires. Nous avons donc décidé d'affecter la surface des exploitations aux ateliers principaux et de réserver aux ateliers secondaires la possibilité d'épandre sur les terres libres du canton.

On fait l'hypothèse que toutes les terres épandables du département sont utilisées pour épandre les effluents d'élevage, à hauteur du plafond défini dans la directive nitrates, soit 170 
$\mathrm{kg}$ d'azote organique par hectare de surface épandable ${ }^{5}$. Cela revient à négliger certains coûts et économies liés à la fertilisation minérale, et à considérer que l'offre de droits d'épandage est rigide et égale à la capacité d'accueil d'effluents maximale.

Les ateliers d'élevage ont le choix entre plusieurs techniques de résorption de l'azote. Les effluents des ateliers principaux peuvent être épandus sur les terres de l'atelier (épandage direct) ou transportés pour fertiliser d'autres terres situées dans le canton, le département ou à l'extérieur de la Bretagne (exportation). Cinq bassins céréaliers situés dans le centre-ouest de la France ont été intégrés au modèle, en prévision des exportations d'effluents avicoles. En revanche, on a considéré qu'il n'y avait pas d'exportation d'effluents vers les départements limitrophes, déjà très chargés en azote organique ${ }^{6}$. Les coûts de transport entre les unités spatiales ont été calculés pour les différents effluents. Le coût de transport de l'unité d'azote porc est plus élevé que celui de l'unité d'azote avicole, car le lisier de porc est trois fois plus riche en eau que les effluents avicoles (tableau 2). Le modèle contraint les ateliers bovins, liés au sol, à épandre systématiquement leurs effluents sur les terres du canton d'origine. Les coûts de transaction associés aux échanges d'effluents n'ont pas été pris en compte.

Tableau 2. Coûts de transport des effluents d'élevage

\begin{tabular}{|c|c|c|c|c|c|c|c|c|c|}
\hline \multirow[b]{2}{*}{ Atelier } & \multirow[b]{2}{*}{$\begin{array}{l}\text { Type } \\
\text { d'effluent }\end{array}$} & \multirow{2}{*}{$\begin{array}{l}\text { Densité du } \\
\text { produit } \\
(\mathrm{kg} \text { d'azote } \\
\left./ \mathrm{m}^{3}\right)\end{array}$} & \multicolumn{7}{|c|}{ Coûts de transport selon la distance ( $€ / \mathrm{kg}$ d'azote) } \\
\hline & & & $20 \mathrm{~km}$ & $50 \mathrm{~km}$ & $100 \mathrm{~km}$ & $200 \mathrm{~km}$ & $300 \mathrm{~km}$ & 400 km & $500 \mathrm{~km}$ \\
\hline Porcins & Lisier & 3,9 & 1,50 & 2,00 & 2,70 & 4,40 & & & \\
\hline $\begin{array}{l}\text { Volailles } \\
\text { chair }\end{array}$ & Fumier & 10,4 & & & 0,41 & 0,72 & 0,69 & 0,86 & 1,02 \\
\hline $\begin{array}{l}\text { Poules } \\
\text { pondeuses }\end{array}$ & $\begin{array}{l}\text { Fientes } \\
\text { séchées }\end{array}$ & 12,0 & & & 0,35 & 0,62 & 0,60 & 0,75 & 0,89 \\
\hline
\end{tabular}

\footnotetext{
${ }^{5}$ On a calculé une surface d'accueil (350000 ha), égale à la surface épandable (70\% de la SAU), additionnée d'une surface équivalente au potentiel de résorption des prairies permanentes par pâturage (100 kg d'azote / ha).

${ }^{6}$ En Bretagne, la réglementation interdit le transfert des effluents vers les cantons à plus de $140 \mathrm{~kg}$ d'azote organique par hectare épandable.
} 
Les effluents peuvent également être traités dans des installations individuelles ou collectives, en utilisant des technologies qui diffèrent selon le type d'élevage. En porc, la technologie individuelle est le traitement biologique du lisier (dénitrification sans séparation de phase). Les ateliers porcs ont également accès à deux usines collectives qui utilisent la technologie de méthanisation-cogénération ${ }^{7}$. Ces usines, qui cristallisent beaucoup d'oppositions, ont été limitées à deux pour tester leur intérêt. Le séchage des fientes de poules pondeuses au niveau de l'atelier diminue les coûts de transport et d'épandage, sans abattement d'azote. Le compostage individuel du fumier de volaille chair est une opération peu coûteuse qui abat la teneur en azote de $30 \%$. En outre, le fumier de volaille chair peut être incinéré dans deux usines collectives. Les usines de cogénération et d'incinération ont été localisées respectivement au sein des zones de forte densité porcine et avicole chair. Le modèle ne prévoit pas le traitement des effluents bovins.

L'azote organique peut également être réduit à la source. L'alimentation «biphase » permet aux élevages de porcs de réduire leurs rejets azotés, en utilisant deux aliments dont le taux azoté est adapté aux besoins du porc, plus élevés en croissance qu'en finition.

Enfin, l'azote peut être résorbé par réduction du cheptel. Le coût de réduction du cheptel a été modulé selon l'horizon temporel. A long terme, on considère que l'éleveur (et ses salariés) peut investir et travailler dans un autre secteur d'activité : le coût est alors donné par le résultat de l'exploitation. A court terme en revanche, les investissements ne sont pas amortis et le marché du travail n'est pas suffisamment fluide : le coût correspond à la marge brute, c'est-à-dire au résultat augmenté des coûts fixes qui comprennent les amortissements, le coût du travail salarié et la rémunération du travail de l'éleveur. Les coûts de production utilisés ici ne comprennent pas les coûts de gestion des effluents, de façon à permettre l'arbitrage entre les différentes solutions de résorption. A court terme, l'éleveur réduit son cheptel quand le coût de la réduction du cheptel est inférieur au coût de résorption par une autre technique, c'est-à-dire quand le prix de vente devient inférieur au coût variable moyen intégrant les coûts de gestion des effluents (seuil de fermeture). Le traitement des effluents est considéré à court terme comme un facteur variable, dans la mesure où la décision d'investir n'a pas encore été prise. A long terme, tous les facteurs sont variables et l'éleveur réduit son cheptel quand le

\footnotetext{
${ }^{7}$ L'usine produit de l'électricité à partir de la combustion de biogaz issu de la méthanisation et de gaz naturel. La chaleur perdue est utilisée pour sécher le lisier de porc.
} 
prix de vente est inférieur au coût moyen, coût de gestion de l'azote compris (seuil de rentabilité).

Le modèle autorise la réduction des cheptels porcin et avicole, mais pas celle du cheptel bovin, en raison des services d'entretien de l'espace qu'il rend et des aides qu'il pourrait recevoir à ce titre. Le tableau 3 donne les principaux coûts (incluant investissement et fonctionnement) et marges moyens ramenés à l'unité d'azote résorbé, qui ont été retenus dans le programme linéaire. L'annexe 1 explicite les modalités de calcul et les sources qui ont servi à élaborer les données des tableaux 2 et 3 . On observe des économies d'échelle dans la production animale et le traitement individuel du lisier de porc.

Tableau 3. Coûts de résorption de l'azote organique

\begin{tabular}{|c|c|c|c|c|c|c|}
\hline \multirow[t]{2}{*}{ Atelier } & \multirow{2}{*}{ Techniques de gestion des effluents } & \multirow{2}{*}{$\begin{array}{l}\text { Taux de } \\
\text { résorption } \\
\text { de l'azote }\end{array}$} & \multicolumn{4}{|c|}{$\begin{array}{l}\text { Coût selon la production d'azote ( } € / \text { kg d'azote } \\
\text { résorbé) }\end{array}$} \\
\hline & & & $\begin{array}{l}<20000 \\
\mathrm{~kg}\end{array}$ & $\begin{array}{l}20000 \text { à } \\
30000 \mathrm{~kg}\end{array}$ & $\begin{array}{l}>30000 \\
\mathrm{~kg}\end{array}$ & $\begin{array}{l}\text { Atelier } \\
\text { secondaire }\end{array}$ \\
\hline \multirow{7}{*}{ Porcins } & Epandage direct du lisier & $100 \%$ & 1,30 & 1,40 & 2,10 & 1,30 \\
\hline & Traitement biologique individuel & $80 \%$ & 5,58 & 4,03 & 3,55 & 4,79 \\
\hline & $\begin{array}{l}\text { Traitement biologique individuel + Epandage } \\
\text { coproduit }\end{array}$ & $100 \%$ & 4,93 & 3,69 & 3,31 & 4,30 \\
\hline & $\begin{array}{l}\text { Traitement par cogénération industrielle + } \\
\text { Epandage coproduit }\end{array}$ & $100 \%$ & 4,15 & 4,15 & 4,15 & 4,15 \\
\hline & Alimentation biphase & $17 \%$ & 0,33 & 0,26 & 0,20 & 0,33 \\
\hline & Réduction du cheptel à Court Terme & $100 \%$ & 12,06 & 12,66 & 14,25 & 11,62 \\
\hline & Réduction du cheptel à Long Terme & $100 \%$ & 1,82 & 2,19 & 4,08 & 2,21 \\
\hline \multirow{5}{*}{$\begin{array}{l}\text { Volailles } \\
\text { chair }\end{array}$} & Epandage direct de fumier & $100 \%$ & 0,89 & 0,91 & 0,97 & 1,05 \\
\hline & Compostage de fumier & $30 \%$ & 0,47 & 0,41 & 0,42 & 0,47 \\
\hline & Incinération industrielle & $100 \%$ & 2,43 & 2,43 & 2,43 & 2,43 \\
\hline & Réduction du cheptel à Court Terme & $100 \%$ & 7,57 & 7,60 & 7,65 & 7,74 \\
\hline & Réduction du cheptel à Long Terme & $100 \%$ & 1,17 & 1,57 & 1,81 & 0,00 \\
\hline \multirow{4}{*}{$\begin{array}{l}\text { Poules } \\
\text { pondeuses }\end{array}$} & Epandage direct de fiente humide & $100 \%$ & 0,62 & 0,62 & 0,63 & 0,72 \\
\hline & Séchage + Epandage & $100 \%$ & 1,10 & 1,32 & 1,76 & 2,73 \\
\hline & Réduction du cheptel à Court Terme & $100 \%$ & 7,87 & 9,72 & 10,92 & 7,05 \\
\hline & Réduction du cheptel à Long Terme & $100 \%$ & 0,64 & 2,49 & 3,70 & 0,00 \\
\hline Bovins & Epandage direct de fumier & $100 \%$ & 1,33 & 1,35 & 1,39 & 1,55 \\
\hline
\end{tabular}


Deux scénarios principaux ont été testés, à court et à long terme. Le premier scénario, qui autorise les échanges d'effluents entre ateliers à l'intérieur des cantons et entre les cantons du département, est qualifié de scénario «marché ». Le deuxième scénario, qui n'autorise pas les échanges entre ateliers, est appelé «sans transfert». Néanmoins, les transferts d'effluents avicoles vers les bassins céréaliers sont possibles dans les deux scénarios. La réalité bretonne se situe entre ces deux scénarios, car seulement $50 \%$ des terres libres à l'épandage par des tiers reçoivent des effluents animaux. Un scénario intermédiaire qui limite les échanges aux transferts à l'intérieur des cantons a été regardé ( «transfert intra »). On a également chiffré le coût du Plan d'action pour un développement pérenne de l'agriculture bretonne. Elaboré par la Préfecture de région et les professionnels en 2002, ce plan fixe les objectifs et les techniques de résorption de l'azote par type d'élevage et par canton (annexe 2). Les instruments imposés par le plan sont le traitement individuel du lisier, l'alimentation biphase des porcs, l'exportation des effluents avicoles et l'incinération du fumier de volailles. Bien que des aides à l'investissement soient prévues, nous avons chiffré le coût réel horssubventions du plan, pour pouvoir le comparer au coût obtenu quand le principe pollueurpayeur est appliqué avec ou sans transfert d'effluents. Les coûts unitaires des tableaux 2 et 3 sont donc inchangés dans les différents scénarios.

On présente d'abord la structure simplifiée du modèle du scénario «marché » (les variables du modèle sont définies au tableau 4) :

$$
\underset{E, B, A, T, U, R}{\operatorname{Min}} \sum_{o} \sum_{e} \sum_{t}\left[\begin{array}{l}
\sum_{d} E_{e t o d}\left(c_{e t o d}^{1}+c_{e t}^{4}\right)+\sum_{k} B_{e t o k}\left(c_{e t o k}^{2}+c_{e}^{4}\right)+A_{e t o} \cdot c_{e t}^{5} \\
+T_{e t o} \cdot c_{e t}^{6}+\sum_{j} U_{e t o j}\left(c_{e t o j}^{3}+c_{e j}^{7}\right)+R_{e t o} \cdot c_{e t}^{8}
\end{array}\right]
$$

Sous les contraintes :

$$
\begin{array}{ll}
E_{\text {etod }} \geq 0 & \forall e, t, o, d \\
B_{\text {etok }} \geq 0 & \forall e, t, o, k \\
A_{\text {eto }} \geq 0 & \forall e, t, o \\
T_{\text {eto }} \geq 0 & \forall e, t, o
\end{array}
$$




$$
\begin{array}{ll}
U_{\text {etoj }} \geq 0 & \forall e, t, o, j \\
R_{\text {eto }} \geq 0 & \forall e, t, o
\end{array}
$$

$$
\sum_{d} E_{e t o d}+\sum_{k} B_{e t o k}+A_{e t o}+T_{e t o}+\sum_{j} U_{e t o j}+R_{e t o}=N_{e t o} \quad \forall e, t, o
$$

$$
\sum_{o} \sum_{e} \sum_{t} U_{e t o j} \leq K_{j} \quad \forall j
$$

$$
\begin{array}{ll}
\sum_{k} B_{\text {etok }} \leq N_{\text {eto }} & \forall e, t, o \\
\sum_{d} E_{\text {etod }} \leq N_{\text {eto }} & \forall e, t, o \\
A_{\text {eto }} \leq N_{\text {eto }} & \forall e, t, o \\
T_{\text {eto }} \leq N_{\text {eto }} & \forall e, t, o \\
\sum_{j} U_{\text {etoj }} \leq N_{\text {eto }} & \forall e, t, o \\
R_{\text {eto }} \leq N_{\text {eto }} & \forall e, t, o
\end{array}
$$

$$
\sum_{o} \sum_{e} \sum_{t} E_{\text {etod }} \leq 170\left(\sum_{e} \sum_{t} S_{\text {etd }}\right) \quad \forall d
$$

La fonction objectif minimisée est le coût de résorption par les différentes techniques, sommé sur les 16 ateliers d'élevage de tous les cantons du département (équation 1). Pour chaque atelier, le coût de résorption est la somme du coût d'épandage et de transport vers les différents cantons et éventuellement l'extérieur du département, de l'alimentation biphase, du coût de traitement individuel et dans les usines collectives le cas échéant, et du coût de la réduction du cheptel à court ou à long terme. Sur la base d'informations données par des sociétés spécialisées dans le transfert d'effluents, on fait l'hypothèse que l'éleveur qui souhaite exporter ses effluents assure ou prend en charge le transport et l'épandage, que ce 
soit dans le département ou dans les bassins céréaliers proches de Bretagne. Les contraintes (2) à (7) imposent la positivité des variables. La contrainte (8) assure que la production d'azote de chaque atelier est effectivement résorbée par les différentes techniques de résorption de l'azote (épandage dans le département ou à l'extérieur, alimentation biphase, traitement individuel ou collectif, réduction du cheptel). La contrainte (9) définit la capacité maximale de traitement des usines collectives. Les contraintes (10) à (15) assurent que chaque atelier ne peut résorber plus que sa production par les différentes méthodes. Enfin, la contrainte (16) garantit que la somme des quantités d'azote provenant des ateliers des différents cantons respecte la contrainte d'épandage de la directive nitrates dans le canton de destination. Le prix fictif associé à cette contrainte donne une estimation de la valeur du droit d'épandage dans chaque canton.

La spécification du modèle du scénario « sans transfert » intègre les restrictions propres à ce scénario :

$\underset{E, B, A, U, R, T}{\operatorname{Min}} \sum_{o} \sum_{e} \sum_{t}\left[\begin{array}{l}E_{e t o o} \cdot c_{e t}^{4}+\sum_{k} B_{e t o k}\left(c_{e t o k}^{2}+c_{e}^{4}\right)+A_{e t o} \cdot c_{e t}^{5} \\ +T_{e t o} \cdot c_{e t}^{6}+\sum_{j} U_{e t o j}\left(c_{e t o j}^{3}+c_{e j}^{7}\right)+R_{e t o} \cdot c_{e t}^{8}\end{array}\right]$

Sous les contraintes (3), (4), (5), (6), (7), (9), (10), (12), (13), (14), (15) et :

$$
\begin{aligned}
& E_{\text {etoo }}+\sum_{k} B_{\text {etok }}+A_{\text {eto }}+T_{\text {eto }}+\sum_{j} U_{\text {etoj }}+R_{\text {eto }}=N_{\text {eto }} \quad \forall e, t, o \\
& E_{\text {etoo }} \leq N_{\text {eto }} \quad \forall e, t, o \\
& E_{\text {etoo }} \leq 170 S_{\text {eto }} \quad \forall e, t, o
\end{aligned}
$$

Les équations (17) et (18) ne prévoient pas d'épandage hors du canton d'origine et la contrainte (20) va plus loin en restreignant l'épandage aux seules terres propres de l'atelier. La contrainte (19) est une contrainte de capacité. 
L'application de programmation utilisée est le module de recherche opérationnelle SAS/OR du logiciel de programmation mathématique SAS. L'algorithme de résolution LPsolve permet de résoudre les programmes linéaires.

Tableau 4. Définition des variables du modèle

\begin{tabular}{|l|l|}
\hline Variable & Définition \\
\hline$E$ & Azote épandu dans le département \\
$B$ & Azote exporté dans un bassin céréalier \\
$A$ & Azote résorbé par alimentation biphase \\
$T$ & azote résorbé par traitement individuel \\
$U$ & Azote résorbé par usine collective \\
$R$ & Azote résorbé par réduction du cheptel \\
$N$ & Azote produit \\
$S$ & Surface épandable \\
$K$ & Capacité de traitement d'une usine collective \\
$C^{1}$ & Coût unitaire de transport de canton à canton \\
$c^{2}$ & Coût unitaire de transport de canton à bassin céréalier \\
$c^{3}$ & Coût unitaire de transport de canton à usine collective \\
$c^{4}$ & Coût unitaire d'épandage \\
$c^{5}$ & Coût unitaire de l'alimentation biphase \\
$c^{6}$ & Coût unitaire de traitement individuel \\
$c^{7}$ & Coût unitaire de traitement collectif \\
$c^{8}$ & Coût unitaire de réduction du cheptel \\
\hline & Canton d'origine \\
\hline & Type d'atelier \\
\hline & Taille de l'atelier \\
\hline
\end{tabular}




\section{Résultats}

Les choix de résorption des élevages de porcs sont présentés au tableau 5. La différence entre les scénarios de court terme et long terme réside dans le recours accru au traitement individuel et collectif du lisier à court terme et à la réduction du cheptel à long terme. Le marché permet de limiter la réduction du cheptel à $20 \%$ des effectifs, soit un niveau proche de celui du plan, qui fait pourtant massivement appel au traitement du lisier. Le traitement du lisier et la réduction du cheptel sont logiquement observés dans les zones où la concentration porcine était initialement forte. Les scénarios «marché» maximisent l'exportation du lisier, qui représente un quart de l'azote produit par les porcs, sans compter les ateliers secondaires. La comparaison des résultats entre les scénarios "sans transfert » et "marché », par classe de taille, montre que le marché permet aux petits ateliers de ne pas traiter leurs effluents à court terme. Dans le même esprit, à long terme, le marché limite la réduction du cheptel aux ateliers les moins efficaces. Globalement, les élevages de porcs contribuent pour la moitié à l'effort de réduction du cheptel dans le département, toutes espèces confondues. L'alimentation biphase est intéressante à court terme, mais pas à long terme en raison de la réduction du cheptel et de la moindre pression sur la terre.

Tableau 5. Gestion de l'azote dans les exploitations porcines selon les scénarios (\% de l'azote porc agrégé)

\begin{tabular}{|l|l|l|l|l|l|l|}
\hline \multirow{2}{*}{ Techniques de gestion } & \multicolumn{4}{l}{ Court Terme } & \multicolumn{2}{l}{ Long Terme } \\
\cline { 2 - 8 } & Sans-transfert & Marché & Plan & Sans-transfert & Marché & Plan \\
\hline Réduction du cheptel & - & - & - & 38,4 & 20,7 & 14,0 \\
\hline Alimentation biphase & 13,9 & 13,5 & 15,2 & 3,9 & 2,7 & 12,8 \\
\hline Epandage direct & 28,9 & 33,5 & 25,8 & 34,3 & 32,5 & 27,4 \\
\hline Traitement individuel & 30,1 & 5,6 & 45,1 & 3,0 & - & 44,7 \\
\hline Traitement collectif & 7,2 & 2,8 & 2,5 & - & - & 0,1 \\
\hline Exportation & - & 26,7 & - & - & 24,5 & - \\
\hline $\begin{array}{l}\text { Epandage des ateliers } \\
\text { secondaires }\end{array}$ & 19,9 & 17,9 & 11,4 & 20,4 & 19,6 & 1,0 \\
\hline
\end{tabular}


Les éleveurs de poules pondeuses privilégient l'exportation de fientes séchées à court terme et réduisent le cheptel à long terme de 20 ou $40 \%$, selon que le plan soit mis en œuvre ou pas (tableau 6). Quand elle est possible, l'exportation de fientes humides sur le marché proche permet de limiter (à court terme) ou d'annuler (à long terme) l'exportation de fientes séchées hors du département.

A court terme, les élevages de volailles chair ont recours à la technologie de compostage couplée à l'exportation des composts, mais moins dans le cas du plan qui impose l'incinération des fumiers (tableau 7). A long terme, la réduction du cheptel diminue la pression sur les terres d'épandage et l'intérêt du compostage. Elle ne dépasse pas $30 \%$ des effectifs quand les transferts d'effluents sont possibles. Bien qu'ils soient en excédent comme les élevages de porcs, les élevages de poules pondeuses et de volailles chair utilisent moins leurs terres pour l'épandage direct que les élevages de porcs. La possibilité d'exporter ou de traiter leurs effluents (voire de réduire leur cheptel) à des coûts avantageux les incite à libérer leurs terres, afin de louer leurs services d'épandage aux élevages de porcs.

Tableau 6. Gestion de l'azote dans les exploitations de poules pondeuses selon les scénarios (\% de l'azote pondeuses agrégé)

\begin{tabular}{|c|c|c|c|c|c|c|}
\hline \multirow[t]{2}{*}{ Techniques de gestion } & \multicolumn{3}{|l|}{ Court Terme } & \multicolumn{3}{|l|}{ Long Terme } \\
\hline & Sans-transfert & Marché & Plan & Sans-transfert & Marché & Plan \\
\hline Réduction du cheptel & - & - & - & 41,2 & 42,2 & 20,6 \\
\hline $\begin{array}{l}\text { Epandage direct fientes } \\
\text { humides }\end{array}$ & 17,7 & 12,0 & 12,5 & 17,2 & 10,3 & 7,4 \\
\hline Exportation fientes humides & - & 37,4 & - & - & 47,5 & - \\
\hline Exportation fientes séchées & 68,8 & 40,3 & 74,0 & 41,6 & - & 72,0 \\
\hline $\begin{array}{l}\text { Epandage des ateliers } \\
\text { secondaires }\end{array}$ & 13,5 & 10,3 & 13,5 & - & - & - \\
\hline
\end{tabular}


Tableau 7. Gestion de l'azote dans les exploitations de volailles de chair selon les scénarios (\% de l'azote volailles chair agrégé)

\begin{tabular}{|l|l|l|l|l|l|l|}
\hline \multirow{2}{*}{ Techniques de gestion } & \multicolumn{5}{l}{ Court Terme } & \multicolumn{3}{l|}{ Long Terme } \\
\cline { 2 - 8 } & Sans-transfert & Marché & Plan & Sans-transfert & Marché & Plan \\
\hline Réduction du cheptel & - & - & - & 66,4 & 28,7 & 35,5 \\
\hline Exportation du fumier & 1,2 & 1,2 & 1,2 & 1,2 & 19,9 & 1,2 \\
\hline $\begin{array}{l}\text { Incinération dans usine } \\
\text { collective }\end{array}$ & - & - & 35,0 & - & - & 35,0 \\
\hline Compostage & 29,6 & 29,6 & 19,1 & 9,7 & 15,4 & 8,5 \\
\hline $\begin{array}{l}\text { Epandage direct } \\
\text { compost }\end{array}$ & 18,8 & 11,2 & 17,6 & 19,0 & 14,4 & 18,8 \\
\hline $\begin{array}{l}\text { Epandage compost } \\
\text { ateliers secondaires }\end{array}$ & 19,9 & 9,9 & 18,3 & 0,2 & 0,2 & 0,2 \\
\hline Exportation compost & 30,5 & 48,0 & 8,7 & 3,5 & 21,4 & 0,8 \\
\hline
\end{tabular}

Le tableau 8 donne des informations sur le lieu, le volume et la nature des échanges d'effluents. Globalement, les effluents excédentaires sont majoritairement transférés vers les terres libres de leur canton d'origine. Ce profil est encore accentué à long terme, en raison de la réduction du cheptel qui diminue le besoin d'exportation. L'exportation de lisier de porc hors du canton d'origine n'est jamais intéressante, à cause de coûts de transport élevés. Les fientes de poule sont exportées dans le département et à l'extérieur uniquement à court terme. En revanche, que ce soit à court ou à long terme, le fumier de volailles est principalement échangé sur le marché inter-cantonal. Les zones qui exportent des effluents (figures 3 et 6) sont les zones à forte concentration animale, situées au centre du département. Les zones qui acceptent des effluents (figures 4 et 7), situées à l'est et à l'ouest, se rapprochent des zones exportatrices à long terme, en raison d'une moins forte tension sur les marchés locaux suite à la réduction du cheptel. Cela se traduit par une réduction de la distance moyenne des transactions, qui passe de 30 à $40 \mathrm{~km}$ à court terme à $15 \mathrm{~km}$ à long terme. Les prix des droits d'épandage ramenés à l'hectare, approchés par le prix fictif de la contrainte azote de chaque canton, sont plus élevés au centre du département (figures 2 et 5). Ils varient entre 61 et 638 euros par hectare à court terme et entre 0 et 148 euros par hectare à long terme. A long terme, les droits n'ont plus de valeur dans les zones où la contrainte azote n'est plus saturée, c'est-àdire à l'est et à l'ouest du département. 


\section{Tableau 8. Transferts d'effluents dans les scénarios « marché »}

\begin{tabular}{|l|l|l|l|l|l|l|}
\hline \multirow{2}{*}{$\begin{array}{l}\text { Horizon et lieu } \\
\text { des transferts }\end{array}$} & $\begin{array}{l}\text { Transferts intra } \\
\text { cantonaux }\end{array}$ & $\begin{array}{l}\text { Transferts } \\
\text { inter } \\
\text { cantonaux }\end{array}$ & $\begin{array}{l}\text { Exportation } \\
\text { hors } \\
\text { département }\end{array}$ & $\begin{array}{l}\text { Transferts intra } \\
\text { cantonaux }\end{array}$ & $\begin{array}{l}\text { Transferts inter } \\
\text { cantonaux }\end{array}$ & $\begin{array}{l}\text { Exportation hors } \\
\text { département }\end{array}$ \\
\hline $\begin{array}{l}\text { Azote transféré } \\
\text { (tonne) }\end{array}$ & 14501 & 4974 & 713 & 14147 & 1256 & - \\
\hline Porc (\%) & 65,4 & 0,0 & 0,0 & 66,2 & 0,0 & - \\
\hline Poule (\%) & 23,5 & 50,7 & 100,0 & 24,0 & 0,0 & - \\
\hline Volaille chair (\%) & 10,3 & 49,3 & 0,0 & 9,0 & 100,0 & - \\
\hline Bovin (\%) & 0,8 & 0,0 & 0,0 & 0,8 & 0,0 & - \\
\hline
\end{tabular}

Comme prévu, le marché permet de réduire significativement le coût de résorption de l'azote des élevages de porcs et de volailles, par rapport aux scénarios «sans transfert » (de $37 \%$ et $23 \%$, à court et long terme respectivement: tableau 9). L'essentiel du gain provient des transactions à l'intérieur des cantons, car les écarts de coût entre scénarios «marché » et «transfert intra» sont très faibles. Ceci est cohérent avec le fait que le marché bénéficie surtout aux élevages de porcs (tableau 9). Le plan d'action breton diminue le recours à l'épandage à court terme et long terme, et la réduction du cheptel à long terme. La différence de coût entre les scénarios «sans transfert» et le plan vient de ce que le plan impose le traitement collectif et individuel, qui est peu intéressant, particulièrement à long terme. Au total, le coût du plan est proche du double de celui du marché. 
Tableau 9. Coût de résorption total et répartition par type d'atelier et par technique de résorption, selon les différents scenarios

\begin{tabular}{|c|c|c|c|c|c|c|c|}
\hline \multirow{2}{*}{ Scénarios } & & \multicolumn{3}{|c|}{ Court terme } & \multicolumn{3}{|c|}{ Long terme } \\
\hline & & Sans-transfert & Marché & Plan & Sans-transfert & Marché & Plan \\
\hline \multicolumn{2}{|c|}{ Coût total $(1000 €)$} & 74245 & 46877 & 85123 & 48053 & 37103 & 80763 \\
\hline \multirow{3}{*}{$\begin{array}{l}\text { Type d'atelier } \\
(\%)\end{array}$} & Porc & 75,3 & 72,7 & 74,6 & 73,3 & 83,0 & 76,8 \\
\hline & Poule & 18,6 & 17,1 & 18,1 & 17,2 & 10,6 & 17,5 \\
\hline & Volaille chair & 6,1 & 10,2 & 7,4 & 9,5 & 6,3 & 5,7 \\
\hline \multirow{7}{*}{$\begin{array}{l}\text { Technique de } \\
\text { résorption (\%) }\end{array}$} & $\begin{array}{l}\text { Réduction du } \\
\text { cheptel }\end{array}$ & - & - & - & 41,2 & 25,4 & 8,4 \\
\hline & $\begin{array}{l}\text { Alimentation } \\
\text { biphase }\end{array}$ & 7,2 & 11,1 & 7,0 & 2,5 & 2,3 & 6,2 \\
\hline & $\begin{array}{l}\text { Epandage } \\
\text { direct }\end{array}$ & 12,4 & 21,5 & 9,5 & 22,3 & 26,8 & 10,3 \\
\hline & $\begin{array}{l}\text { Epandage des } \\
\text { ateliers } \\
\text { secondaires }\end{array}$ & 8,9 & 12,1 & 4,9 & 11,7 & 14,6 & 0,3 \\
\hline & $\begin{array}{l}\text { Traitement } \\
\text { individuel }\end{array}$ & 48,7 & 18,0 & 59,4 & 13,0 & 1,2 & 59,1 \\
\hline & $\begin{array}{l}\text { Traitement } \\
\text { collectif }\end{array}$ & 8,0 & 4,9 & 8,5 & - & - & 6,5 \\
\hline & $\begin{array}{l}\text { Exportation } \\
\text { d'effluent }\end{array}$ & 14,8 & 32,4 & 10,7 & 9,3 & 29,8 & 9,2 \\
\hline
\end{tabular}

\section{Discussion}

Notre modèle avait pour objectif de simuler l'adaptation des élevages aux contraintes de la directive nitrates, dans un département de l'ouest de la France très chargé en animaux. Les élevages de porcs et de poules choisissent le traitement ou l'exportation à court terme, et réduisent leur cheptel respectivement de $20 \%$ et $40 \%$ à long terme. Les élevages de volailles chair compostent et exportent leurs effluents vers les autres cantons, et réduisent leurs effectifs de $30 \%$ à long terme. Le marché diminue le recours au traitement et à l'exportation des effluents hors du département, ainsi que la réduction du cheptel, en optimisant l'épandage direct. Dans le secteur porcin, le marché incite les élevages les plus efficaces à traiter leurs effluents à court terme, et les élevages les moins efficaces à réduire leur cheptel à long terme. Les échanges à long terme ont principalement lieu à l'intérieur des cantons et concernent les 
élevages de porcs surtout et les élevages de poules. Le prix des droits à long terme varie entre 0 et 150 euros par hectare. A long terme, le marché permet de réduire le coût de résorption de l'azote des porcs et des volailles d'environ $20 \%$ par rapport à une situation sans échanges et de la moitié par rapport au plan d'action régional, réduction qui concerne surtout le secteur porcin.

L'étude montre que les différentes productions animales adoptent des stratégies spécifiques, en fonction de la rentabilité, du coût de transport et du coût de traitement de l'unité d'azote. A long terme, le traitement des effluents d'élevage n'est pas rentable, à l'exception du compostage. Les élevages privilégient la réduction du cheptel et l'épandage, c'est-à-dire la liaison au sol. En incitant certains élevages à réduire leur cheptel et à louer leurs droits d'épandage à d'autres éleveurs, le marché régule la concentration animale. Les prix de location des droits que nous obtenons sont proches du prix du fermage, ce qui permet d'anticiper une hausse substantielle du prix du foncier, qui a déjà commencé en Bretagne (Le Goffe et Salanié, 2005).

Les prix des droits sont élevés à court terme, car les alternatives à la location des terres d'épandage sont coûteuses, tout particulièrement la réduction du cheptel qui n'est jamais choisie. Le scénario de court terme suppose que les éleveurs font appel à des réserves financières, des emprunts ou des subventions qui leur permettent de continuer à produire tant que les bâtiments d'élevage ne sont pas amortis. Dans le cas contraire, certains éleveurs font faillite et le scénario de long terme arrive rapidement. La subvention peut être couplée à la mise en œuvre d'une technique de résorption, par exemple le traitement des effluents. Dans ce cas, la baisse du coût perçu par les éleveurs encourage le recours à la technique aidée et se traduit par des prix plus bas sur le marché de l'épandage. La diminution de la tension sur les marchés de l'épandage et de la terre, favorable à l'installation de nouveaux éleveurs, est d'ailleurs un argument avancé par les professionnels pour légitimer l'aide au traitement du lisier. Ici, la concentration animale n'est plus régulée, ce qui rejoint l'effet d'expansion de la branche d'une subvention à la dépollution, évoqué par Baumol et Oates (1988).

C'est cette option qui a été choisie dans le Plan d'action pour un développement pérenne de l'agriculture bretonne, dans le but d'aider les éleveurs en difficulté et d'éviter la réduction du cheptel. Cette solution contraire au principe pollueur-payeur ne peut être que transitoire et on peut se demander si il ne faudrait pas lui préférer une aide à la réduction du cheptel, comme cela se pratique aux Pays-Bas et en Flandres belges, où une baisse du cheptel pour cause environnementale a été observée. Mahé et Le Goffe (2002) ont ainsi proposé une aide 
« neutre » qui ne modifie pas la décision - de traiter ou de réduire son cheptel - que prendrait l'éleveur à long terme en l'absence de subvention. Alors que l'agro-alimentaire et le tourisme sont les deux grands secteurs économiques de Bretagne, la réduction du cheptel provoquerait à court terme une baisse de la valeur ajoutée régionale et de l'emploi, qui a été étudiée dans le cas des Pays-Bas (Komen et Peerlings, 1998). Au-delà de l'aide aux éleveurs, il faudrait probablement accompagner l'aval des filières agro-alimentaires pendant la phase d'ajustement structurel, avec l'objectif de développer la valeur ajoutée avec moins de production animale (élaboration des produits, développement des labels et des marques, typicité régionale, etc...).

Le travail présente également plusieurs limites. Certains coûts n'ont pas été pris en compte : coûts de transport des effluents à l'intérieur des cantons, coûts de transaction, coûts de substitution de l'azote organique à l'azote minéral. En conséquence, le modèle surestime les échanges et les économies permises par le marché, et sous-estime le recours au traitement ou à la réduction du cheptel. Cependant, vu que la distance maximale à l'intérieur d'un canton est de $20 \mathrm{~km}$ et compte tenu des coûts unitaires de transport et de résorption, on montre facilement que l'absence de coûts de transport intra-cantonaux n'affecte pas les choix de résorption à court terme, mais peut en revanche minorer la réduction du cheptel à long terme. Nous avions envisagé d'étudier les échanges entre des zones communales. Ce choix aurait alourdi considérablement le modèle et posait en outre des problèmes de secret statistique. Une autre solution aurait été de considérer des coûts moyens de transport intra-cantonaux.

En Bretagne, les expériences d'organisation de l'épandage commencent à apparaître, stimulées notamment par le contentieux en cours avec l'Union Européenne. Le GIE (Groupement d'Intérêt Economique) «Terre-eau » rassemble plusieurs éleveurs, localisés dans 8 cantons d'Ille et Vilaine tous classés en ZES, qui souhaitent transférer leurs excédents de lisier vers des cantons en déficit d'amendements organiques. Le projet, qui a bénéficié de l'appui technique de la Chambre d'agriculture, a demandé un travail important pour recenser les agriculteurs ayant des disponibilités en plan d'épandage, obtenir leur accord et réaliser les études préalables à l'agrément du plan d'épandage collectif par l'Administration. Il a rencontré une forte opposition des communes destinées à recevoir les excédents de lisier, allant jusqu'à la contestation de la décision d'autorisation du Préfet devant le tribunal administratif. Les coûts d'organisation unitaires sont probablement importants ici. Des transactions de gré à gré entre agriculteurs provoqueraient probablement moins d'opposition des populations et moins de difficultés administratives qu'un projet collectif de grande ampleur nécessitant une planification et une autorisation. 
L'hypothèse de stabilité du cheptel bovin est probablement trop forte. L'évolution des cheptels dépend de la rentabilité relative par unité d'azote. Les bovins ne seraient pas nécessairement épargnés, comme le montre l'exemple du Danemark où le cheptel porc progresse au détriment du cheptel bovin, à azote constant. Cette question se pose d'autant plus que les marchés et les politiques agricoles sont entrés dans une période de turbulences depuis 2003. Enfin, nous avons travaillé à partir de la structure actuelle de la production animale, en autorisant les réductions mais pas les augmentations de cheptel. Le relâchement de cette hypothèse conduirait à une relocalisation de l'élevage, avec des créations ou extensions d'élevage dans les zones à faible densité, qui ne pourraient plus accueillir d'effluents en provenance des zones surchargées, où les réductions du cheptel seraient alors plus importantes que celles que nous observons. Il en résulterait l'uniformisation de la densité animale et des prix des droits d'épandage et du foncier, comme cela a été observé aux PaysBas (Vukina et Wossink, 2000).

Certaines améliorations du modèle pourraient facilement être entreprises, comme l'introduction de coûts de transaction (voir Pujol et al., 2006), ou d'une norme d'épandage en phosphore. Bien qu'il n'y ait pas encore de directive européenne sur le phosphore, il existe en France des réglementations régionales sur l'épandage des coproduits du traitement des effluents, qui sont riches en phosphore. Ribaudo et al. (2003) signalent qu'il faut plus de terres pour respecter une norme phosphore que pour respecter une norme azote, car le rapport phosphore/azote des effluents d'élevage est plus élevé que celui du besoin des plantes. La norme phosphore induirait donc une tension sur les marchés de l'épandage et une réduction du cheptel plus importante, d'autant plus que le traitement technologique du phosphore est plus coûteux que celui de l'azote.

Une amélioration beaucoup plus complexe consisterait à mesurer la structure des profits, coûts de traitement et coûts de substitution marginaux par unité d'azote, ce qui supposerait un travail économétrique d'envergure pour estimer ces fonctions d'offre et de demande de droits d'épandage. Une autre voie, utilisable notamment pour estimer l'offre de droits d'épandage, serait de coupler un modèle agronomique à un modèle économique. Lanzanova (2007) a ainsi estimé une fonction de demande d'eau d'irrigation en résolvant par la programmation mathématique un modèle de maximisation du profit du producteur agricole couplé au modèle agronomique STICS.

Les mesures arrêtées récemment par la France sur les bassins versants bretons en contentieux ont convaincu la Commission Européenne de suspendre temporairement son action en justice. 
Les éleveurs devront respecter des normes de fertilisation totale par culture et par système de production (140 kg d'azote par hectare pour le système grandes cultures / élevages hors-sol). Les pertes de revenu liées à la baisse de rendement des cultures et au surcoût de la gestion des effluents seront compensées par des aides temporaires et dégressives sur 5 ans, sous forme de mesures agro-environnementales volontaires d'abord, puis d'indemnité compensatoire de contrainte environnementale à partir de 2008. Des aides équilibrées entre le traitement et le transfert des effluents d'une part, la réduction des cheptels d'autre part, sont également prévues. Les amendes pour dépassement des normes de fertilisation ne sont pas évoquées, mais elles sont probablement implicites. Avec ce plan d'action, la politique française se rapproche de celles conduites dans les pays du nord de l'Europe, au Danemark, en Flandres belges et aux Pays-Bas (voir Fermin, 2007), même si la mise en oeuvre du principe pollueurpayeur reste encore floue et retardée. Un marché de l'épandage actif devrait donc se développer à terme sur ces bassins versants en contentieux. Il reste que ce plan d'action, qui mobilisera 86 millions d'euros sur 5 ans, ne concerne que $4 \%$ de la surface agricole bretonne. Le reste du territoire breton n'est donc pas à l'abri d'un nouveau dérapage des effectifs animaux, si les marchés sont favorables et si la politique des bassins versants en contentieux n’est pas généralisée. 


\section{Références}

Baron, P., Barthélémy, F., Bouvier, M., Martin, X., Vogler, J.P. (2001). Elevages et fonctionnement du Conseil Départemental d'Hygiène en Ille et Vilaine. Ministère de l'aménagement du territoire et de l'environnement, Paris.

Baumol, W.J., Oates, W.E. (1988). The Theory of Environmental Policy. Cambridge University Press, Cambridge.

Feinerman, E., Komen, M.H.C. (2005). The use of organic vs. chemical fertilizer with a mineral losses tax: the case of Dutch arable farmers. Environmental and Resource Economics, 32: 367-388.

Fermin, N. (2007). Application de la directive «nitrates » dans trois pays européens : PaysBas, Danemark, Flandres. Rapport sous la direction de P. Le Goffe pour la Préfecture de Région Bretagne, 89 p.

Helming, J.F.M. (1998). Effects of nitrogen input and nitrogen surplus taxes in Dutch agriculture. Cahiers d'Economie et Sociologie Rurales, 49: 6-31.

Kaplan, J.D., Johansson, C. Peters, M. (2004). The manure hits the land: economic and environmental implications when land application of nutrients is constrained. American Journal of Agricultural Economic, 86(3): 688-700.

Keplinger, K.O., Hauck, L.M. (2006). The economics of manure utilization: model and application. Journal of Agricultural and Resource Economics, 31(2): 414-440.

Komen, M.H.C., Peerlings, J.H.M. (1998). Restricting intensive livestock production: Economic effects of mineral policy in The Netherlands. European Review of Agricultural Economics, 25: 110-128.

Lanzanova, D. (2007). How irrigation rationing affect water value. Presentation at the European Association of Agricultural Economists PhD Workshop: Economic Research in Food, Agriculture, Environment and Development. September 4-5, Rennes.

Le Goffe, P., Salanié, J. (2005). Le droit d'épandage a-t-il un prix ? Mesure sur le marché foncier. Cahiers d'Economie et Sociologie Rurales, 77: 36-63.

Le Goffe, P. (2005). Le projet de loi sur l'eau : une réflexion économique. In : Evolution de la qualité des eaux en nitrate. Recommandations pour une nouvelle politique de l'eau. Rapport du Conseil Scientifique de l'Environnement de Bretagne : 62-66. 
Mahé, L.P., Le Goffe, P. (2002). La résorption des excédents d'épandage: principes économiques d'un plan d'action pour la Bretagne. Rapport à la Préfecture de la Région Bretagne : $63 \mathrm{p}$.

Pujol, J., Raggi, M., Viaggi, D. (2006). The potential impact of markets for irrigation water in Italy and Spain: a comparison of two study areas. Australian Journal of Agricultural and Resource Economics, 50(3): 361-380.

Rainelli, P., Vermersch, D. (2000). Gestion foncière des excédents azotés et marché implicite de droits à polluer : application aux élevages intensifs. Economie et Prévision, 143/144(23): 91-100.

Reyns, L., Peeters, L., Veenendaal, P. (1999). Manure in Flanders : assessing the economic consequences of alternative abatement policies. In Agriculture and the environment challenges and conflicts for the new millennium, edited by Turner S.T.D. and Alford D. ADAS Wolverhampton: 93-100.

Ribaudo, M., Kaplan, J., Christensen, L., Gollehon, N., Johansson, R., Breneman, V., Aillery, M., Agapoff, J., Peters, M. (2003). Manure management for water quality: cost to animal feeding operations of applying manure nutrients to land. Agricultural Economic Report $\mathrm{n}^{\circ} 824$, USDA Economic Research Service, Washington DC.

Ribaudo, M., Cattaneo, V., Agapoff, J. (2004). Cost of meeting manure nutrient application standards in hog production: the roles of EQIP and fertilizer offsets. Review of Agricultural Economics, 26(4): 430-444.

Smith, E.G., Card, G., Young, D.L. (2006). Effects of market and regulatory changes on livestock manure management in southern Alberta. Canadian Journal of Agricultural Economics, 54: 199-213.

Vukina, T., Wossink, A. (2000). Environmental policies and agricultural land values: evidence from the Dutch nutrient quota system. Land Economics, 76(3): 413-429. 
Figure 1. Charge en azote organique des élevages en 2000 (kg par hectare)

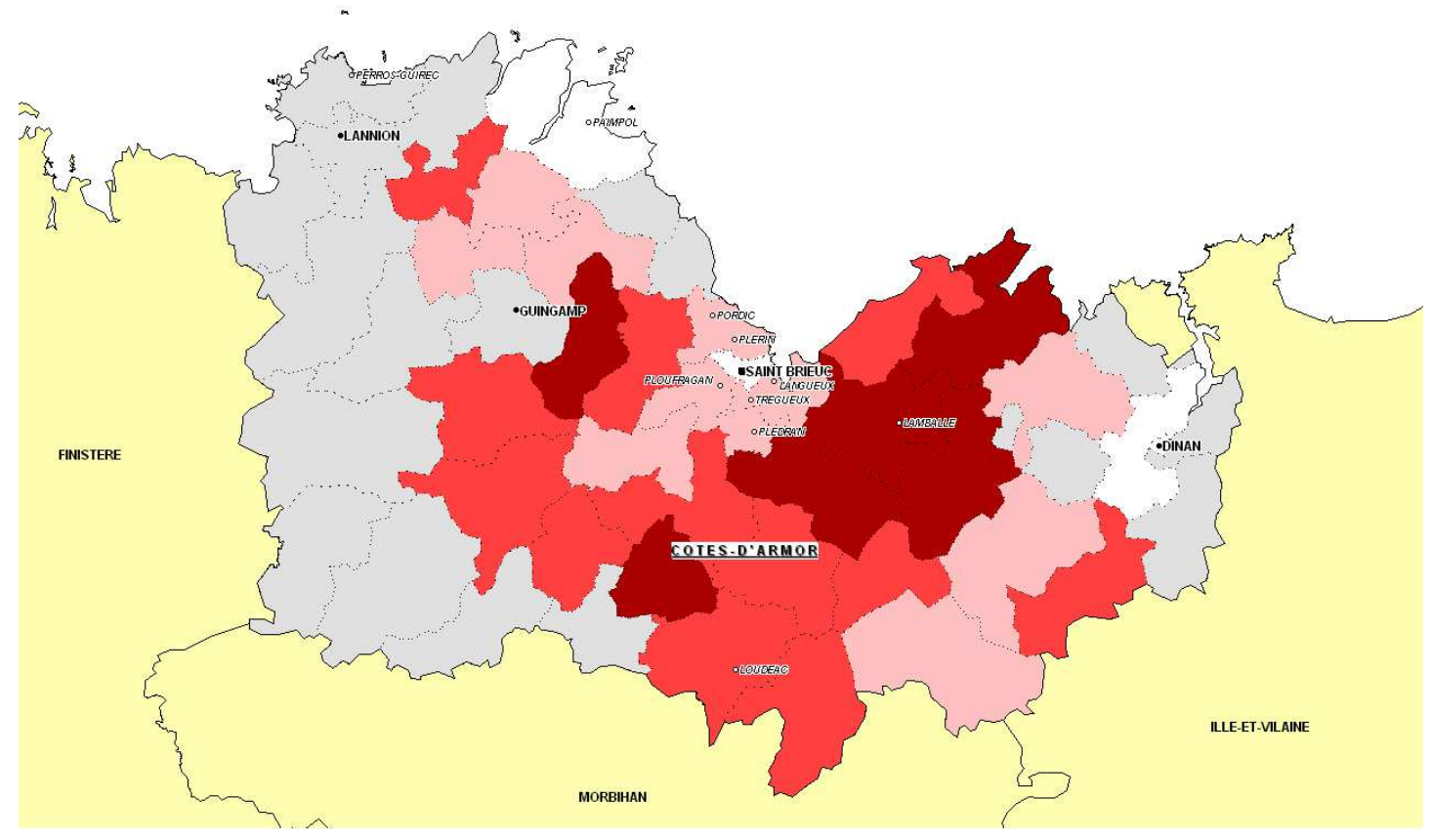

supérieure à 250

entre 200 et 250

Moyenne : 178

entre 170 et 200

Ecart type : 56

entre 120 et 170

intérieure à 120 
Figure 2. Prix des droits d'épandage à court terme (euro par hectare)

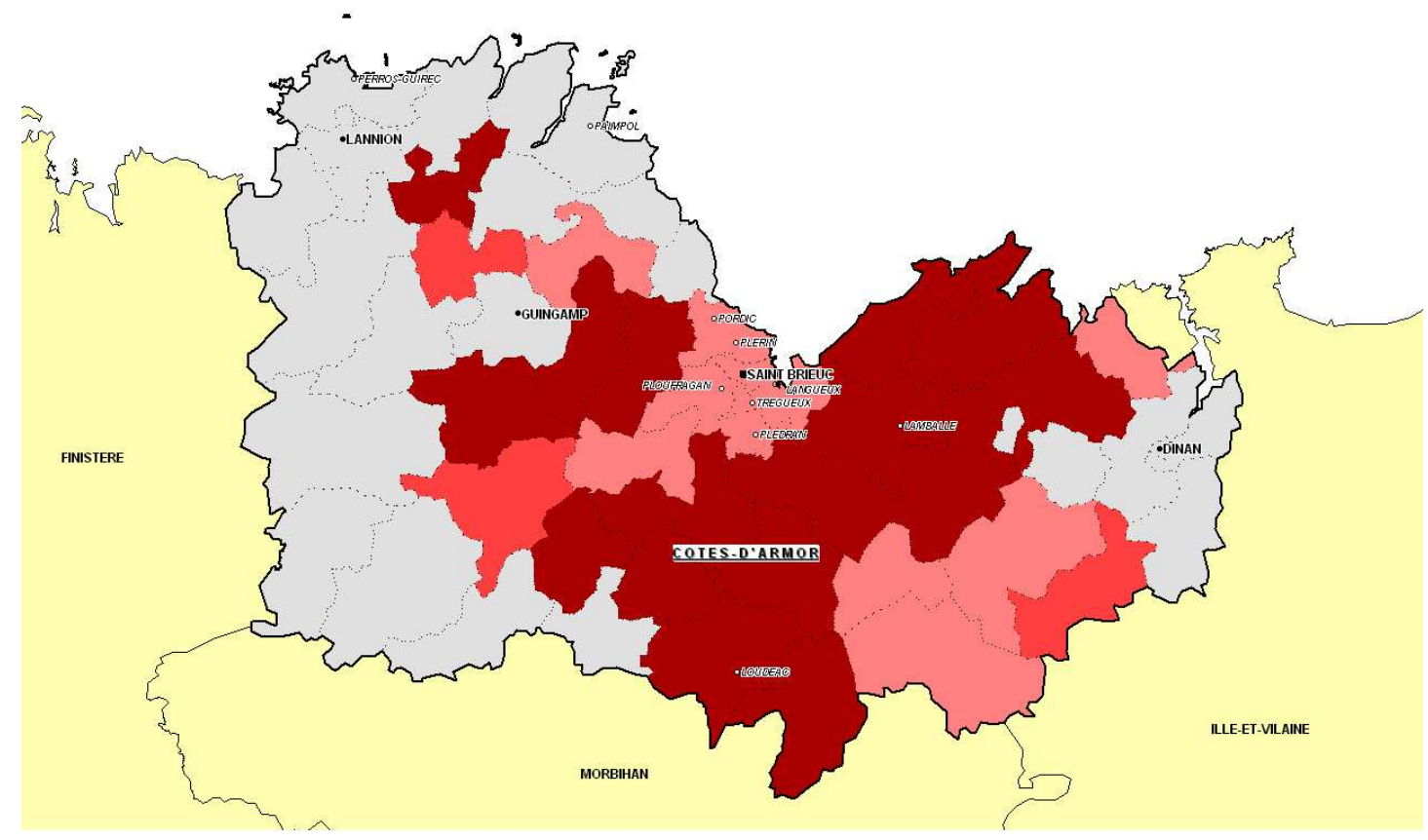

Supérieur à 300

entre 200 et 300

Moyenne : 214

entre 100 et 200

Ecart type : 175

entre 50 et 100 
Figure 3. Cantons exportateurs d'effluents à court terme

(en équivalent hectare à $170 \mathrm{~kg}$ d'azote)

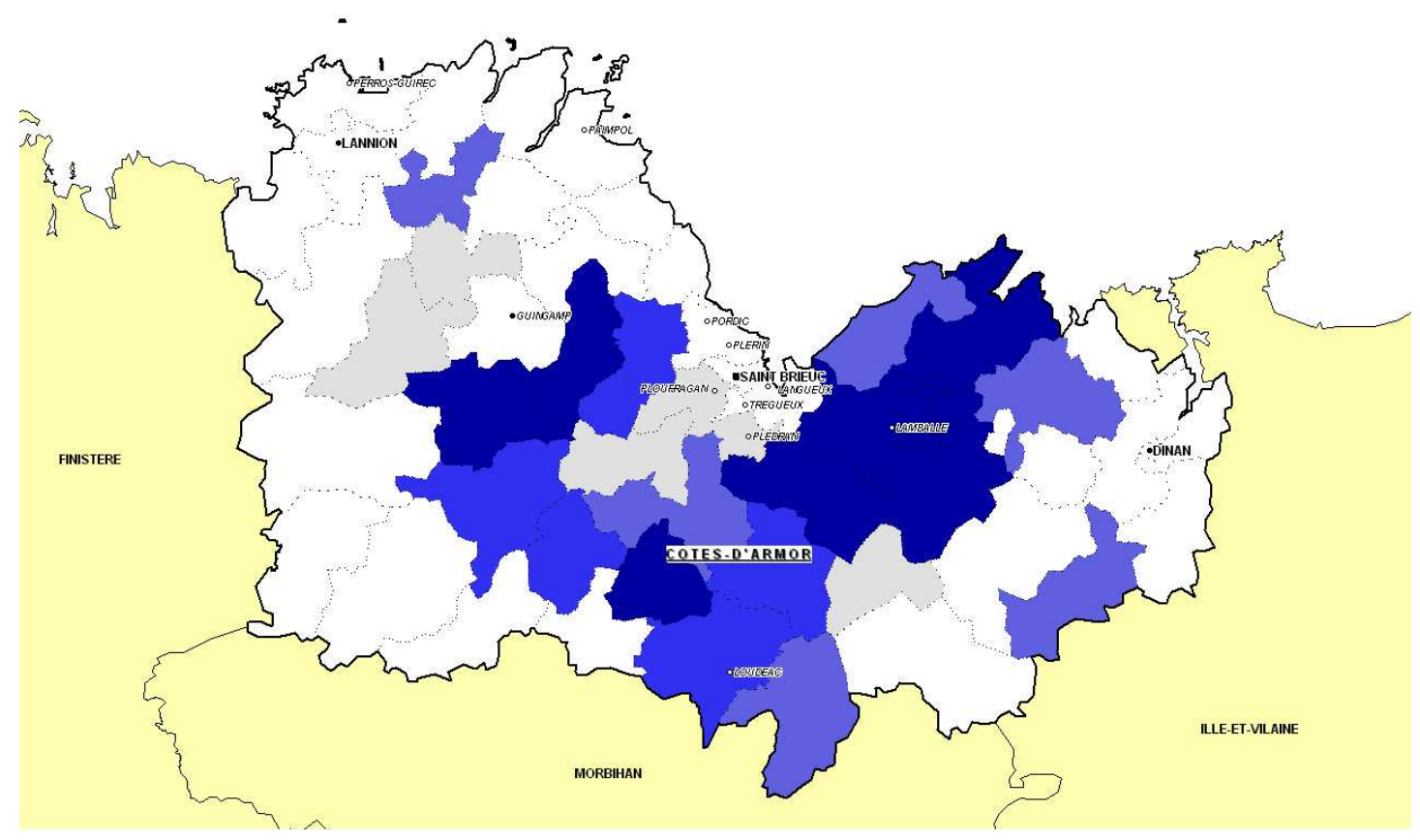

supérieur à 2000

entre 1000 et 2000

entre 500 et 1000

entre 100 et 500

inférieur à 100
Moyenne : 642

Ecart type : 1042 
Figure 4. Cantons récepteurs d'effluents à court terme

(en équivalent hectare à $170 \mathrm{~kg}$ d'azote)

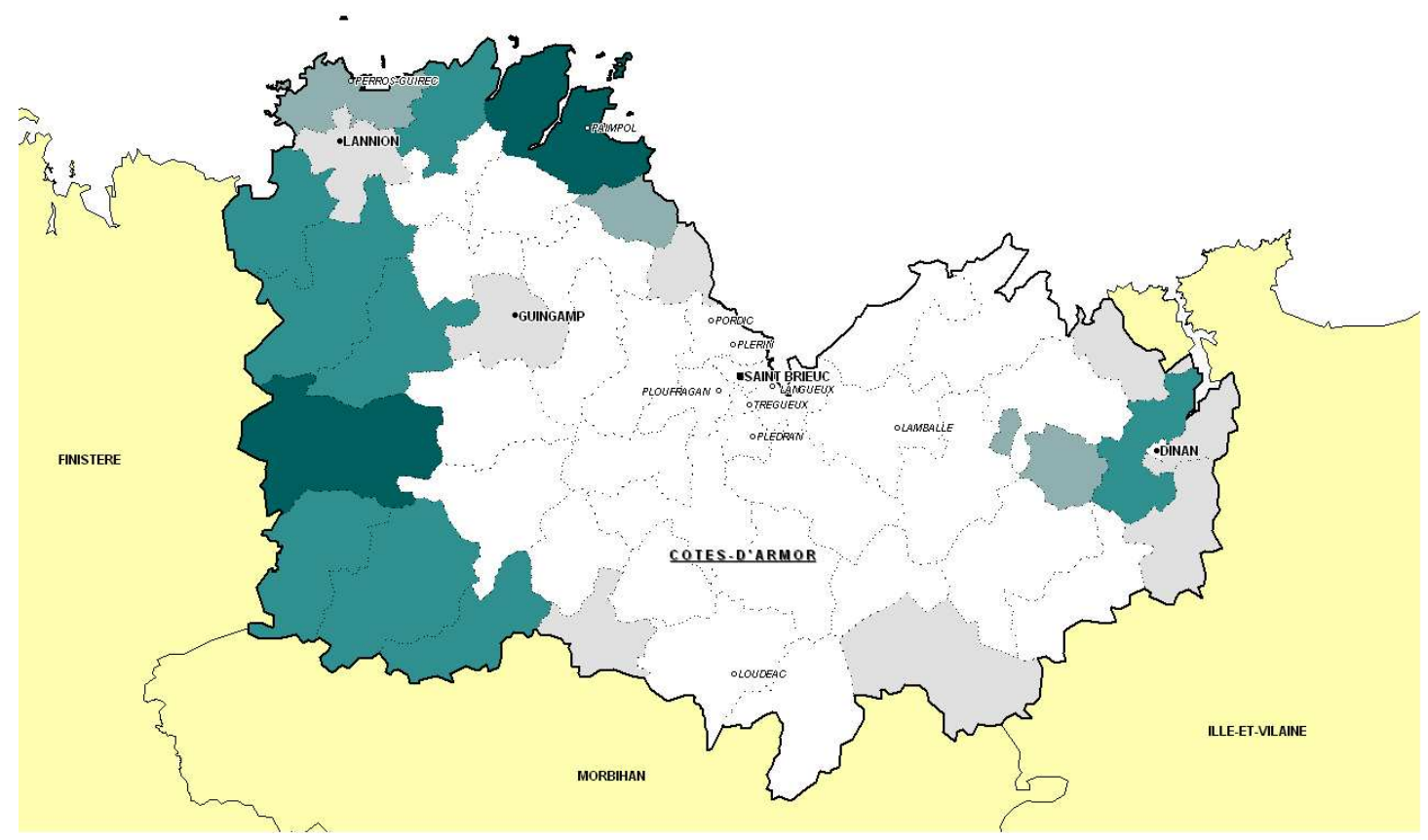

supérieur à 2000

Moyenne : 438

entre 1000 et 2000

Ecart type : 1042

entre 500 et 1000

entre 100 et 500

inférieur à 100 
Figure 5. Prix des droits d'épandage à long terme (euro par hectare)

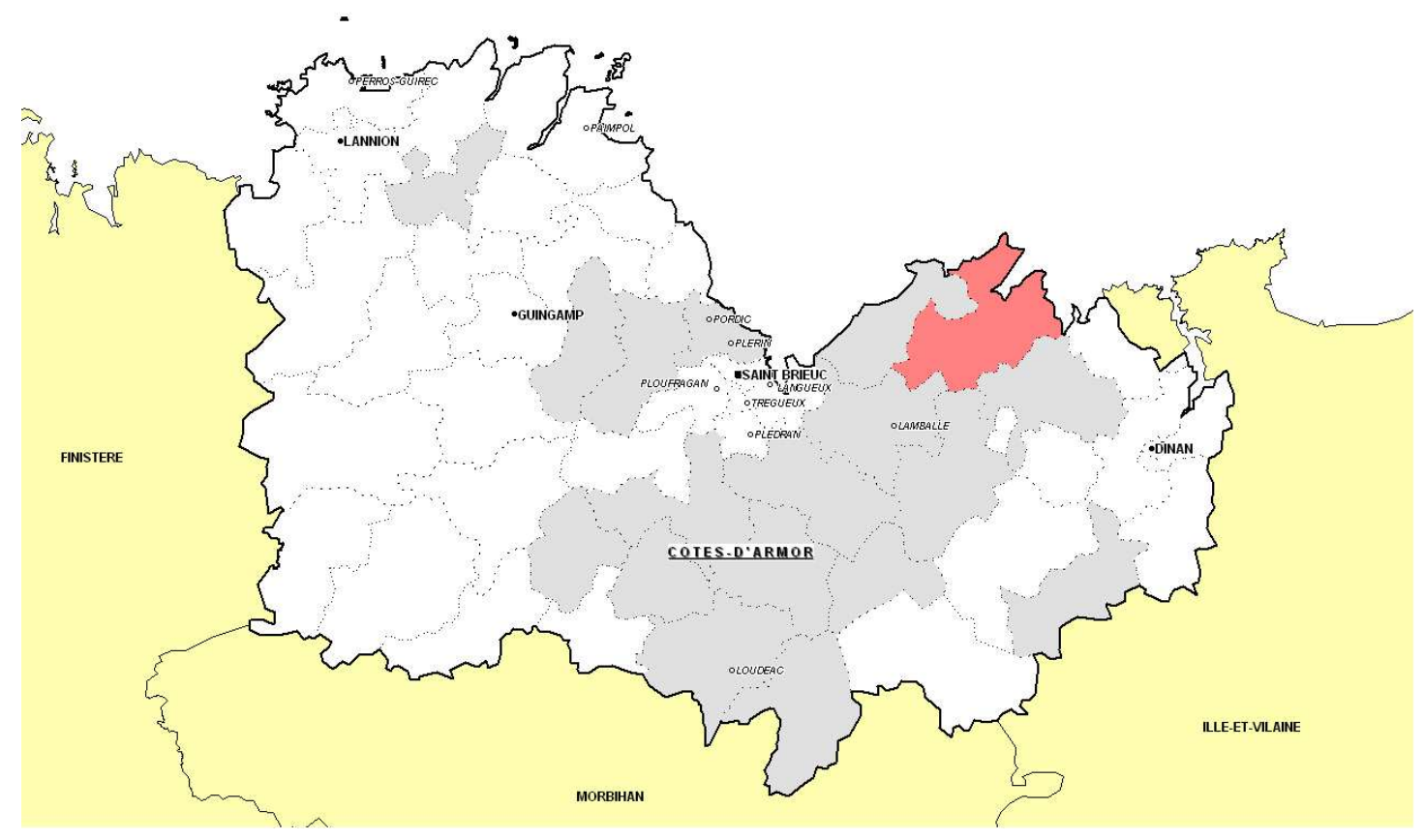

supérieur à 300

entre 200 et 300

Moyenne : 35

entre 100 et 200

entre 50 et 100

inférieur à 50 
Figure 6. Cantons exportateurs d'effluents à long terme

(en équivalent hectare à $170 \mathrm{~kg}$ d'azote)

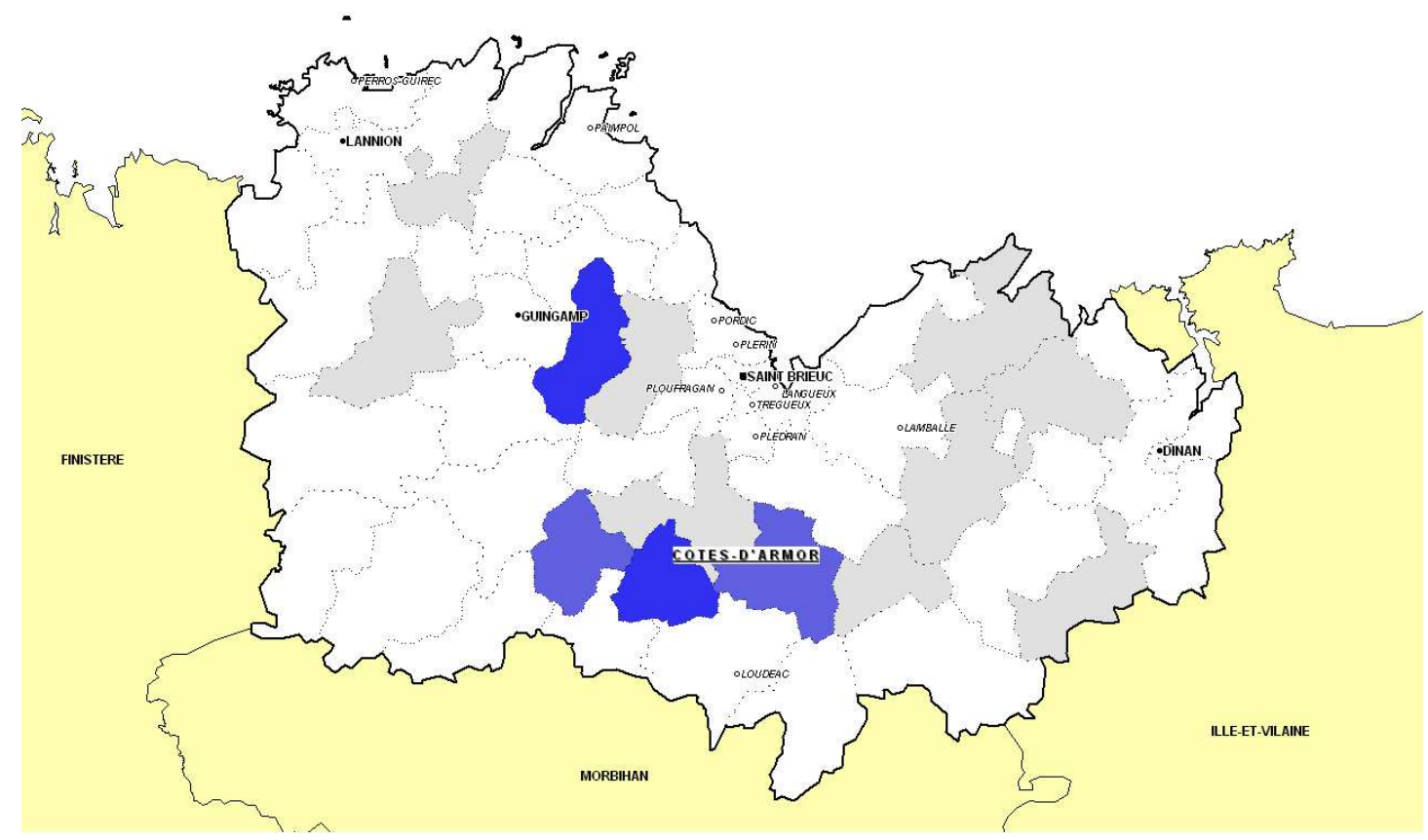

supérieur à 2000

entre 1000 et 2000

Moyenne : 145

entre 500 et 1000

entre 100 et 500

inférieur à 100 
Figure 7. Cantons récepteurs d'effluents à long terme

(en équivalent hectare à $170 \mathrm{~kg}$ d'azote)

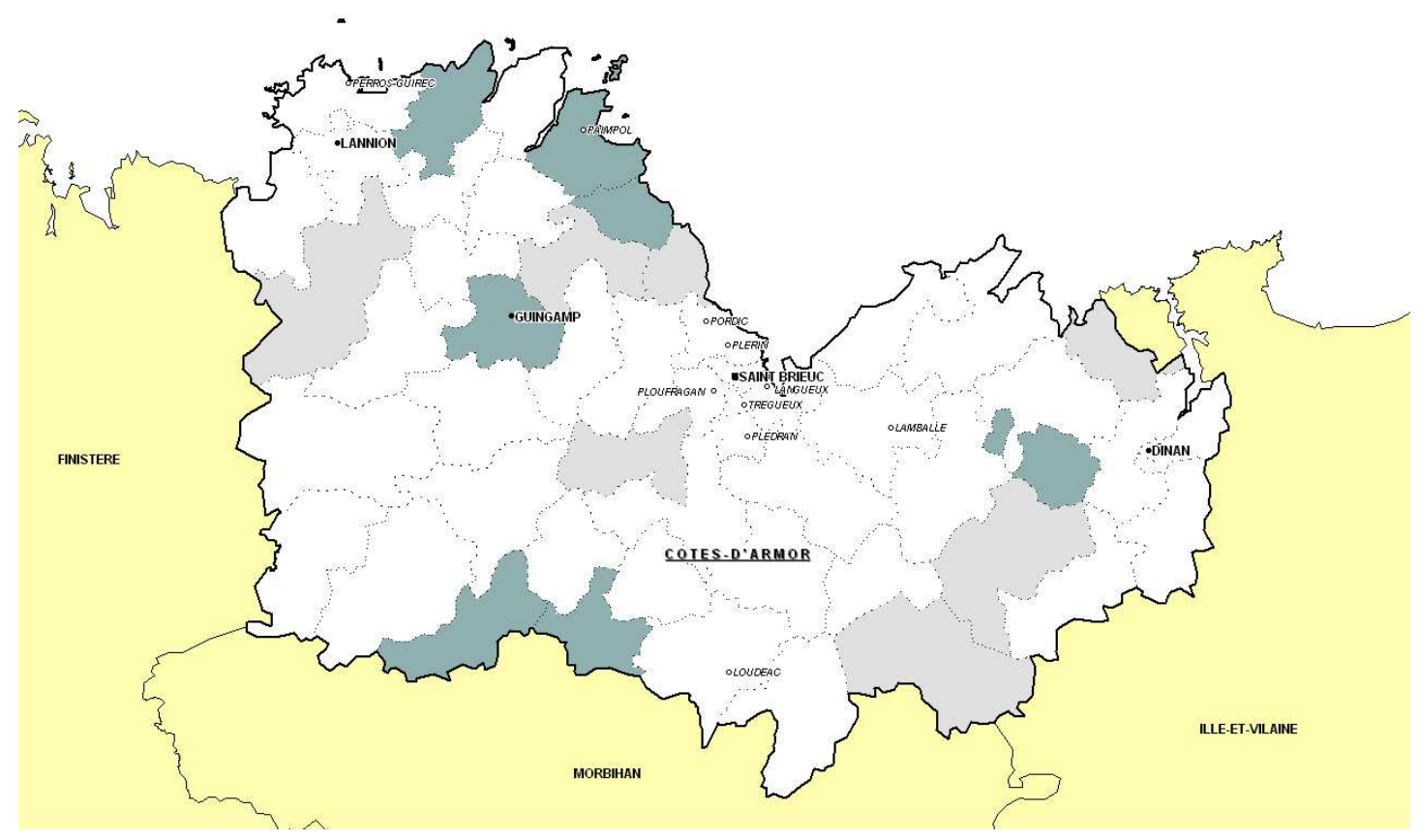

supérieur à 2000

Moyenne : 169

entre 1000 et 2000

Ecart type : 283

entre 500 et 1000

entre 100 et 500

inférieur à 100 
Annexe 1. Sources et modalités de calcul des coûts de transport et de résorption de l'azote

\section{Coûts de transport}

(a) Les coûts de transport reportés dans le tableau 2 ont été calculés sur la base d'une enquête ponctuelle réalisée en 2004 par la Chambre d'agriculture des Côtes d'Armor auprès d'entreprises de transport routier. Le calcul tient compte du temps d'utilisation du camion et de son chauffeur, ainsi que du kilométrage parcouru par le convoi. Ces informations permettent d'établir selon le type de produit transporté, une relation spécifique entre coût de transport d'une unité d'azote entre 2 exploitations et la distance séparant les 2 exploitations.

(b) L'enquête de la Chambre d'agriculture présente une logistique différente pour le transport d'effluents avicoles selon que le trajet dépasse ou non les $200 \mathrm{~km}$. Pour une distance inférieure à $200 \mathrm{~km}$, le retour du camion s'effectue à vide alors que pour un parcours supérieur à $200 \mathrm{~km}$, le camion est lavé et désinfecté pour un retour à plein.

(c) Les coûts de transport de lisier au-delà des $200 \mathrm{~km}$ n'ont pas été présentés car l'enquête de la Chambre d'agriculture révèle que le transport de lisier sur longue distance n'est jamais réalisé, faute de rentabilité économique. La relation entre le coût de transport du lisier et la distance parcourue a donc été établie à partir des données de l'enquête sur des distances inférieures à $200 \mathrm{~km}$. Néanmoins, le modèle n'interdit aucunement le transport de lisier sur de plus longues distances. 
2. Coûts de résorption.

(a) Sources :

\begin{tabular}{|c|c|}
\hline Epandage & $\begin{array}{l}\text { - Etudes de l'Agence de l'eau Loire Bretagne (2000-2004) } \\
\text { - Barème entraide } 2004\end{array}$ \\
\hline $\begin{array}{l}\text { Traitement individuel } \\
\text { du lisier }\end{array}$ & - Etudes de l'Agence de l'eau Loire Bretagne (2000-2004) \\
\hline $\begin{array}{l}\text { Compostage de } \\
\text { fumier de volaille }\end{array}$ & $\begin{array}{l}\text { - Barème entraide } 2004 \\
\text { - Chambre d'agriculture du Morbihan }\end{array}$ \\
\hline Séchage des fientes & - Chambre d'agriculture du Morbihan \\
\hline $\begin{array}{l}\text { Traitement collectif } \\
\text { du lisier }\end{array}$ & $\begin{array}{l}\text { - SENER: Groupe espagnol spécialisé notamment dans le traitement } \\
\text { des déchets }\end{array}$ \\
\hline $\begin{array}{l}\text { Incinération } \\
\text { industrielle de fumier }\end{array}$ & - Chambre d'agriculture du Finistère \\
\hline $\begin{array}{l}\text { Alimentation } \\
\text { biphasée du porc }\end{array}$ & $\begin{array}{l}\text { - Techni porc Vol. 25, No4 - } 2002 \text { (ITP) } \\
\text { - Institut Technique du Porc (ITP) }\end{array}$ \\
\hline Réduction du cheptel & $\begin{array}{l}\text { - Centre Economie Rurale des Côtes d'Armor 2003, 2004, } 2005 \\
\text { - ITP (Résultats } 2003 \text { GTE et GTTT) } \\
\text { - Institut Technique de l'Aviculture }\end{array}$ \\
\hline
\end{tabular}

(b) Les coûts de gestion des effluents comprennent le coût d'investissement matériel, le coût d'investissement immobilier et les coûts de fonctionnement (consommation d'électricité, entretien, réparation, main d'œuvre, consommation de matière première). Les coûts sont annualisés et ramenés à l'unité d'azote résorbée. Pour les investissements, on calcule l'annuité constante équivalente sur la durée de vie du projet, soit 7 ans pour le matériel et 15 ans pour l'immobilier (fosse, station de traitement, etc), à un taux de $6 \%$ par an. 
(c) Les résultats comptables des exploitations d'élevage distinguent le coût opérationnel, le coût de l'outil (amortissement, location, entretien), les coûts financier, du travail salarié et divers (eau, gaz, EDF), les charges sociales et le coût du travail de l'exploitant.

(d) Les prix moyens sont calculés sur des périodes qui diffèrent selon les ateliers, de manière à prendre en compte les cycles ou les modifications durables des prix (porc : 19922004 ; œufs : 2000-2004 ; viande : 1999-2005). En volaille chair, la rentabilité est celle d'une exploitation moyenne qui mélange les différentes productions. 
Annexe 2. Objectifs du Plan d'action pour un développement pérenne de l'agriculture et de l'agroalimentaire et pour la reconquête de la qualité de l'eau en Bretagne

\begin{tabular}{|c|c|c|c|c|c|}
\hline \multirow{2}{*}{$\begin{array}{l}\text { Objectif de résorption (tonne } \\
\text { d'azote) }\end{array}$} & \multicolumn{4}{|c|}{ Départements } & \multirow{2}{*}{ Bretagne } \\
\hline & 22 & 29 & 35 & 56 & \\
\hline Total concernant les ZES & 17797 & 15108 & 3210 & 7797 & 44912 \\
\hline - dont passage au biphase & 2673 & 2314 & 851 & 881 & 6719 \\
\hline $\begin{array}{l}\text { - dont stockage et transfert à } \\
\text { distance de fientes de poules } \\
\text { pondeuses }\end{array}$ & 5017 & 1644 & 116 & 1326 & 8103 \\
\hline - dont traitement de lisier de porc & 7673 & 7981 & 1934 & 2023 & 19611 \\
\hline $\begin{array}{l}\text { - dont incinération et combustion } \\
\text { fumier de volailles }\end{array}$ & 2127 & 2697 & 636 & 3364 & 8824 \\
\hline
\end{tabular}

\begin{tabular}{|l|l|l|l|}
\hline \multicolumn{3}{|l|}{ Objectifs de résorption cantonaux du département 22 (kg d'azote) } \\
\hline Bégard & 269795 & Merdrignac & 400223 \\
\hline Belle-Isle- En-Terre & 118316 & Moncontour & 1146509 \\
\hline Bourbriac & 712846 & Mur-De-Bretagne & 141024 \\
\hline Broons & 432492 & Plancoet & 470723 \\
\hline Caulnes & 414232 & Plelan-Le-Petit & 49681 \\
\hline Chatelaudren & 570097 & Pleneuf-Val-Andre & 556900 \\
\hline Cheze (La) & 637961 & Plerin & 137561 \\
\hline Collinee & 355324 & Ploeuc-Sur-Lie & 491062 \\
\hline Corlay & 690915 & Plouagat & 739492 \\
\hline Dinan-Est & 54856 & Plouaret & 138203 \\
\hline Etables-Sur-Mer & 59975 & Ploubalay & 75686 \\
\hline Evran & 158161 & Ploufragan & 187199 \\
\hline Guingamp & 133016 & Plouguenast & 688095 \\
\hline Jugon-Les-Lacs & 996471 & Pontrieux & 202876 \\
\hline Lamballe & 1414356 & Quintin & 377161 \\
\hline Langueux & 111348 & Roche Derrien (La) & 462877 \\
\hline Lannion & 78186 & Rostrenen & 310676 \\
\hline Lanvollon & 210346 & Saint-Nicolas-Du-Pelem & 646792 \\
\hline Loudeac & 772270 & Uzel & 787299 \\
\hline Mael Carhaix & 297821 & Total & $\mathbf{1 7 7 9 7 3 9 7}$ \\
\hline Matignon & 1298574 & & \\
\hline
\end{tabular}




\section{Les Working Papers SMART - LERECO sont produits par I'UMR SMART et I'UR LERECO}

\section{- UMR SMART}

L'Unité Mixte de Recherche (UMR 1302) Structures et Marchés Agricoles, Ressources et Territoires comprend l'unité de recherche d'Economie et Sociologie Rurales de I'INRA de Rennes et le département d'Economie Rurale et Gestion d'Agrocampus Ouest.

Adresse :

UMR SMART - INRA, 4 allée Bobierre, CS 61103, 35011 Rennes cedex

UMR SMART - Agrocampus, 65 rue de Saint Brieuc, CS 84215, 35042 Rennes cedex

http://www.rennes.inra.fr/smart

\section{- LERECO}

Unité de Recherche Laboratoire d'Etudes et de Recherches en Economie Adresse :

LERECO, INRA, Rue de la Géraudière, BP 7162744316 Nantes Cedex 03

http://www.nantes.inra.fr/le_centre_inra_angers_nantes/inra_angers_nantes_le_site_de_nantes/les_unites/et udes_et_recherches_economiques_lereco

\section{Liste complète des Working Papers SMART - LERECO :}

http://www.rennes.inra.fr/smart/publications/working_papers

\section{The Working Papers SMART - LERECO are produced by UMR SMART and UR LERECO}

\section{- UMR SMART}

The "Mixed Unit of Research" (UMR1302) Structures and Markets in Agriculture, Resources and Territories, is composed of the research unit of Rural Economics and Sociology of INRA Rennes and of the Department of Rural Economics and Management of Agrocampus Ouest.

Address:

UMR SMART - INRA, 4 allée Bobierre, CS 61103, 35011 Rennes cedex, France

UMR SMART - Agrocampus, 65 rue de Saint Brieuc, CS 84215, 35042 Rennes cedex, France http://www.rennes.inra.fr/smart eng/

\section{- LERECO}

Research Unit Economic Studies and Research Lab

Address:

LERECO, INRA, Rue de la Géraudière, BP 7162744316 Nantes Cedex 03, France

http://www.nantes.inra.fr/nantes eng/le centre inra angers nantes/inra angers nantes le site de nantes/l es_unites/etudes_et_recherches_economiques_lereco

Full list of the Working Papers SMART - LERECO:

http://www.rennes.inra.fr/smart_eng/publications/working_papers

\section{Contact}

Working Papers SMART - LERECO

INRA, UMR SMART

4 allée Adolphe Bobierre, CS 61103

35011 Rennes cedex, France

Email : smart_lereco_wp@rennes.inra.fr 
2008

Working Papers SMART - LERECO

UMR INRA-Agrocampus Ouest SMART (Structures et Marchés Agricoles, Ressources et Territoires) UR INRA LERECO (Laboratoires d'Etudes et de Recherches Economiques)

Rennes, France 